\title{
An Extention of Herman's Theorem for Nonlinear Circle Maps with Two Breaks
}

\author{
A. Dzhalilov, D. Mayer, S. Djalilov, A. Aliyev
}

M. Herman showed that the invariant measure $\mu_{h}$ of a piecewise linear (PL) circle homeomorphism $h$ with two break points and an irrational rotation number $\rho_{h}$ is absolutely continuous iff the two break points belong to the same orbit. We extend Herman's result to the class $\mathrm{P}$ of piecewise $C^{2+\varepsilon}$-circle maps $f$ with an irrational rotation number $\rho_{f}$ and two break points $a_{0}, c_{0}$, which do not lie on the same orbit and whose total jump ratio is $\sigma_{f}=1$, as follows: if $\mu_{f}$ denotes the invariant measure of the $P$-homeomorphism $f$, then for Lebesgue almost all values of $\mu_{f}\left(\left[a_{0}, c_{0}\right]\right)$ the measure $\mu_{f}$ is singular with respect to Lebesgue measure.

Keywords: piecewise-smooth circle homeomorphism, break point, rotation number, invariant measure

Received September 10, 2018

Accepted November 19, 2018

Akhtam Dzhalilov

a_dzhalilov@yahoo.com

Turin Polytechnic University

Kichik Halka yuli 17, Tashkent, 100095 Uzbekistan

Dieter Mayer

dieter.mayer@tu-clausthal.de

Institut für Theoretische Physik

TU Clausthal, D-38678 Clausthal-Zellerfeld, Germany

Shukhrat Djalilov

d_shuxrat@mail.ru

Samarkand Institute of Economics and Service

A. Temura st. 9, Samarkand, 140100 Uzbekistan

Abdurakhmon Aliyev

aliyev95@mail.ru

National University of Uzbekistan

VUZ Gorodok, Tashkent, 700174 Uzbekistan

RUSSIAN JOURNAL OF NONLINEAR DYNAMICS, 2018, 14(4), 553-577 


\section{Introduction}

One of the important problems of circle dynamics is that of absolute continuity of the invariant measure for circle homeomorphisms. For smooth circle homeomorphisms this problem is well understood. Fundamental results on this were obtained by V. Arnold, M. Herman, J. Moser, J.-C. Yoccoz, Ya. Sinai and K. Khanin, Y. Kaznelson and D. Ornstein and others. Let $f$ be an orientation-preserving homeomorphism of the circle $S^{1} \equiv \mathbb{R} / \mathbb{Z}$ with lift $F: \mathbb{R} \rightarrow \mathbb{R}$, which is continuous, strictly increasing and fulfills $F(\widehat{x}+1)=F(\widehat{x})+1, \widehat{x} \in \mathbb{R}$. The circle homeomorphism $f$ is then defined by $f(x)=F(\widehat{x}) \bmod 1$ with $\widehat{x} \in \mathbb{R}$ a lift of $x \in S^{1}$. The rotation number $\rho_{f}$ is defined by

$$
\rho_{f}:=\lim _{n \rightarrow \infty} \frac{F^{n}(\widehat{x})-\widehat{x}}{n} \bmod 1,
$$

where $F^{i}$ denotes the $i$ th iteration of the map $F$. It is well known that the rotation number $\rho_{f}$ does not depend on the starting point $\widehat{x} \in \mathbb{R}$ and is irrational if and only if $f$ has no periodic points (see [4]). The rotation number $\rho_{f}$ is invariant under topological conjugations.

Denjoy's classical theorem states [5] that a circle diffeomorphism $f$ with an irrational rotation number $\rho=\rho_{f}$ and $\log D f$ of bounded variation can be conjugated to the linear rotation $R_{\rho}$ with lift $\widehat{R}_{\rho}(\widehat{x})=\widehat{x}+\rho$, that is, there exists a homeomorphism $\varphi: S^{1} \rightarrow S^{1}$ with $f=\varphi \circ R_{\rho} \circ \varphi^{-1}$.

It is well known that a circle homeomorphism $f$ with an irrational rotation number $\rho_{f}$ is uniquely ergodic, i.e., it has a unique invariant probability measure $\mu_{f}$. A remarkable fact then is that the conjugacy $\varphi$ can be defined by $\varphi(x)=\mu_{f}([0, x])$ (see [4]), which shows that the smoothness properties of the conjugacy $\varphi$ imply corresponding properties of the density of the absolutely continuous invariant measure $\mu_{f}$ for a sufficiently smooth circle diffeomorphism with a typical irrational rotation number (see $[2,12,14,16])$. The problem of smoothness of the conjugacy for smooth diffeomorphisms is by now very well understood (see, for instance, $[2,12,14,16,23])$.

A natural generalization of circle diffeomorphisms are piecewise smooth homeomorphisms with break points (see [14]).

The class of $\mathbf{P}$-homeomorphisms consists of orientation-preserving circle homeomorphisms $f$ which are differentiable except at a finite or countable number of break points, denoted by $B P(f)=\left\{x_{b} \in S^{1}\right\}$, at which the one-sided positive derivatives $D f_{-}$and $D f_{+}$exist, but do not coincide, and for which there exist constants $0<c_{1}<c_{2}<\infty$ such that

- $c_{1}<D f_{-}\left(x_{b}\right)<c_{2}$ and $c_{1}<D f_{+}\left(x_{b}\right)<c_{2}$;

- $c_{1}<D f(x)<c_{2}$ for all $x \in S^{1} \backslash B P(f)$;

- $\log D f$ has finite total variation $v$ in $S^{1}$.

Piecewise linear (for short $P L$ ) orientation-preserving circle homeomorphisms are the simplest examples of $P$-homeomorphisms. They occur in many different areas of mathematics such as group theory, homotopy theory or logic via the Thompson groups. A family of $P L$-homeomorphisms was first studied by M. Herman [14] as examples of circle homeomorphisms with an arbitrary irrational rotation number which admit no invariant $\sigma$-finite measure absolutely continuous with respect to Lebesgue measure.

Indeed, Herman proved thereby

Theorem 1. The invariant measure of a PL-circle homeomorphism with two break points and an irrational rotation number is absolutely continuous with respect to Lebesgue measure if and only if these break points lie on the same orbit. 
Herman's family of maps has been studied later by several authors (see, for instance, [3, 1921]) in the context of interval exchange transformations. Special cases are affine 2-interval exchange transformations, to which Herman's examples with break points $a_{0}=0$ and $c_{0}=c$ belong.

In [8] Dzhalilov et al. proved the following result. Let $f$ be a $P$-homeomorphism with two break points. If $f$ is $C^{2}$ except at the two break points and its rotation number is of bounded type, then the unique $f$-invariant probability measure $\mu_{f}$ is equivalent to Lebesgue measure if and only if the two break points of $f$ lie on the same orbit and its total jump ratio is $\sigma_{f}=1$. Notice that the condition of bounded type is essential: if $\rho_{f}$ is not of bounded type, the homeomorphism $f$ obtained by conjugating a $C^{2}$ diffeomorphism with singular invariant measure (such diffeomorphisms exist by [13]) with a $P$-homeomorphism with one break point is a $P$-homeomorphism with two break points on the same orbit and with a trivial total jump ratio, but the $f$-invariant probability measure $\mu_{f}$ is singular.

The invariant measures of $P$-homeomorphisms $f$ with a finite number of break points have been studied by several authors (see, for instance, $[1,3,6-9,11,15,22]$ ). For such a homeomorphism the character of the invariant measure strongly depends on its total jump ratio $\sigma_{f}$ being trivial or nontrivial, i.e., $\sigma_{f}=1$ or $\sigma_{f} \neq 1$. A recent result of Dzhalilov et al. in [11] in the case $\sigma_{f} \neq 1$ is

Theorem 2. Let $f \in C^{2+\varepsilon}\left(S^{1} \backslash\left\{a_{1}, a_{2}, \ldots, a_{m}\right\}\right)$, $\varepsilon>0$ be a P-homeomorphism with an irrational rotation number $\rho_{f}$ and a finite number of break points $a_{1}, a_{2}, \ldots, a_{m}$. Suppose its total jump ratio is $\sigma_{f}=\sigma_{f}\left(a_{1}\right) \cdot \sigma_{f}\left(a_{2}\right) \cdot \ldots \cdot \sigma_{f}\left(a_{m}\right) \neq 1$. Then its invariant probability measure $\mu_{f}$ is singular with respect to Lebesgue measure $l$.

Piecewise smooth $P$-homeomorphisms $f$ with a finite number of break points and the trivial total jump ratio $\sigma_{f}=1$ are more difficult to investigate. In the special case of piecewise $C^{2+\varepsilon}$ $P$-homeomorphisms $f$ whose break points all lie on the same orbit, the invariant measure $\mu_{f}$ is absolutely continuous w.r.t. to Lebesgue measure for typical irrational rotation numbers (see [7]). Rather complicated is the case where the break points of such a homeomorphism $f$ are not on the same orbit. In this case A. Teplinskii constructed in [22] examples of $P L$-homeomorphisms $f$ with four break points and the trivial total jump ratio $\sigma_{f}=1$ whose irrational rotation numbers $\rho_{f}$ are of unbounded type and whose invariant measures $\mu_{f}$ are absolutely continuous w.r.t. Lebesgue measure $l$.

In the present paper we study $C^{2+\epsilon} P$-homeomorphisms $f$ with an arbitrary irrational rotation number $\rho_{f}$ and two break points not on the same orbit, whose total jump ratio $\sigma_{f}=1$. Put

$$
\begin{aligned}
\Gamma:=\{\rho \in[0,1]: \rho= & {\left[k_{1}, k_{2}, \ldots, k_{n}, \ldots\right] \in \mathbb{R} \backslash \mathbb{Q}: \text { there exists } N=N(\rho) \in \mathbb{N}, } \\
& \text { such that } \left.k_{n} \geqslant 3, \text { for all } n \geqslant N\right\} .
\end{aligned}
$$

Our main result for these homeomorphisms is

Theorem 3. Let $f \in C^{2+\varepsilon}\left(S^{1} \backslash\left\{b_{1}, b_{2}\right\}\right)$ be a P-homeomorphism with an irrational rotation number $\rho:=\rho_{f} \in \Gamma$ and two break points $b_{1}, b_{2}$ on different orbits with the trivial total jump ratio $\sigma_{f}=\sigma_{f}\left(b_{1}\right) \cdot \sigma_{f}\left(b_{2}\right)=1$. Denote its invariant measure by $\mu_{f}$. Then there exists a subset $M_{\rho} \subset[0,1]$ of full Lebesgue measure, such that $\mu_{f}$ is singular w.r.t. Lebesgue measure if $\mu_{f}\left(\left[b_{1}, b_{2}\right]\right) \in M_{\rho}$.

Notice that, if $\mu_{f}\left(\left[b_{1}, b_{2}\right]\right)=q \rho+p, p, q \in \mathbb{Z}$, then the two break points $b_{1}, b_{2}$ lie on the same orbit and the invariant measure $\mu_{f}$ is absolutely continuous w.r.t. Lebesgue measure (see [14]). 
REMARK 1. To every piecewise smooth circle homeomorphism $f$ with two break points $b_{1}, b_{2}$, total trivial jump $\sigma_{f}:=\sigma_{f}\left(b_{1}\right) \cdot \sigma_{f}\left(b_{2}\right)=1$ and an irrational rotation number $\rho:=\rho_{f}$ there corresponds a unique $P L$-map of Herman with two break points $a_{0}=0, c_{0}$, such that $\sigma_{h}\left(a_{0}\right)=\sigma_{f}\left(b_{1}\right)$ and $\mu_{h}\left(\left[a_{0}, c_{0}\right]\right)=$ $=\mu_{f}\left(\left[b_{1}, b_{2}\right]\right)$ (for details see Section 6). Moreover, $\mu_{h}\left(\left[a_{0}, c_{0}\right]\right)=\frac{\beta}{1+\beta}$. Hence, the condition in Theorem 3 can be written also in terms of the parameter $\beta$ of this unique $P L$-map as $\frac{\beta}{1+\beta} \in M_{\rho}$.

REMARK 2. Theorem 3 can be extended to piecewise smooth homeomorphisms of the circle with finitely many break points and a trivial total jump ratio.

Remark 3. The condition $\rho:=\rho_{f} \in \Gamma$ in Theorem 3 is a technical one, and the theorem can be proved for all irrational rotation numbers.

\section{Some definitions and well-known facts}

Let $f$ be an orientation-preserving circle homeomorphism with an irrational rotation number $\rho_{f}$ and an invariant measure $\mu_{f}$. Then $\rho_{f}$ has a unique continued fraction expansion as $\rho_{f}=1 /\left(k_{1}+1 /\left(k_{2}+\ldots\right)\right):=\left[k_{1}, k_{2}, \ldots, k_{n}, \ldots\right)$. Denote by $p_{n} / q_{n}=\left[k_{1}, k_{2}, \ldots, k_{n}\right], n \geqslant 1$, its $n$th convergent. The numbers $q_{n}, n \geqslant 1$, are called also the first return times of $f$ and satisfy the recurrence relations $q_{n+1}=k_{n+1} q_{n}+q_{n-1}, n \geqslant 1$, where $q_{0}=1$ and $q_{1}=k_{1}$. Fix an arbitrary $x_{0} \in S^{1}$. Its forward orbit $O_{f}^{+}\left(x_{0}\right)=\left\{x_{i}=f^{i}\left(x_{0}\right), i=0,1,2 \ldots\right\}$ defines a sequence of natural partitions of the circle. Namely, denote by $I_{0}^{(n)}\left(x_{0}\right)$ the closed interval in $S^{1}$ with endpoints $x_{0}$ and $x_{q_{n}}=f^{q_{n}}\left(x_{0}\right)$. In the clockwise orientation of the circle the point $x_{q_{n}}$ is for $n$ odd to the left of $x_{0}$, and for $n$ even to its right. Denote the iterates of the interval $I_{0}^{(n)}\left(x_{0}\right)$ under $f$ by $I_{i}^{(n)}\left(x_{0}\right):=f^{i}\left(I_{0}^{(n)}\left(x_{0}\right)\right), i \geqslant 1$. We call these intervals for fixed $n$ intervals of rank $n$. If

$$
\Delta_{n}:=\mu_{f}\left(I_{0}^{(n)}\left(x_{0}\right)\right)=\mu_{f}\left(I_{i}^{(n)}\left(x_{0}\right)\right)
$$

denotes the measure of the intervals of rank $n$, then $\Delta_{n}=\left|q_{n} \rho_{f}-p_{n}\right|$.

It is well known that the set $\xi_{n}\left(x_{0}\right)$ of intervals with mutually disjoint interiors, defined as

$$
\xi_{n}\left(x_{0}\right)=\left\{I_{0}^{(n-1)}\left(x_{0}\right), I_{1}^{(n-1)}\left(x_{0}\right), \ldots, I_{q_{n}-1}^{(n-1)}\left(x_{0}\right)\right\} \cup\left\{I_{0}^{(n)}\left(x_{0}\right), I_{1}^{(n)}\left(x_{0}\right), \ldots, I_{q_{n-1}-1}^{(n)}\left(x_{0}\right)\right\},
$$

determines a partition of the circle for any $n$. The partition $\xi_{n}\left(x_{0}\right)$ is called the $n$th dynamical partition of $S^{1}$ determined by the point $x_{0}$ and the map $f$, (where we suppressed the dependence on the map $\mathrm{f}$ for simplicity).

Proceeding from $\xi_{n}\left(x_{0}\right)$ to $\xi_{n+1}\left(x_{0}\right)$, all the intervals $I_{j}^{(n)}\left(x_{0}\right), 0 \leqslant j \leqslant q_{n-1}-1$, are preserved, whereas each of the intervals $I_{i}^{(n-1)}\left(x_{0}\right), 0 \leqslant i \leqslant q_{n}-1$, is partitioned into $k_{n+1}+1$ subintervals belonging to $\xi_{n+1}\left(x_{0}\right)$ such that

$$
I_{i}^{(n-1)}\left(x_{0}\right)=I_{i}^{(n+1)}\left(x_{0}\right) \cup \bigcup_{s=0}^{k_{n+1}-1} I_{i+q_{n-1}+s q_{n}}^{(n)}\left(x_{0}\right) .
$$

Obviously, one has $\xi_{1}\left(x_{0}\right) \leqslant \xi_{2}\left(x_{0}\right) \leqslant \ldots \leqslant \xi_{n}\left(x_{0}\right) \leqslant \ldots$

Definition 1. Let $K>1$ be a constant. We call two intervals $I_{1}$ and $I_{2}$ of $S^{1} K$-comparable if the inequality $K^{-1} \ell\left(I_{2}\right) \leqslant \ell\left(I_{1}\right) \leqslant K \ell\left(I_{2}\right)$ holds. 
Next we introduce another partition $\xi_{n}^{*}\left(x_{0}\right)$. Define

$$
J_{i}^{(n)}:=I_{i}^{(n)}\left(x_{0}\right) \cup I_{i}^{(n-1)}\left(x_{0}\right), \quad 0 \leqslant i \leqslant q_{n-1}-1 .
$$

The system of intervals

$$
\xi_{n}^{*}\left(x_{0}\right):=\left\{J_{0}^{(n)}, J_{1}^{(n)}, \ldots, J_{q_{n-1}-1}^{(n)}\right\} \bigcup\left\{I_{q_{n-1}}^{(n-1)}, I_{q_{n-1+1}}^{(n-1)}, \ldots, I_{q_{n}-1}^{(n-1)}\right\}
$$

determines also a partition of the circle.

Definition 2. An interval $I=[\tau, t] \subset S^{1}$ is said to be $q_{n}$-small and its endpoints $q_{n}$-close if the intervals $f^{i}(I), 0 \leqslant i \leqslant q_{n}-1$, are, except for the endpoints, pairwise disjoint.

Let $f$ be a $P$-homeomorphism with an irrational rotation number $\rho_{f}$ and $|B P(f)|<\infty$. The following facts about $P$-homeomorphisms are standard:

- if the interval $I=(x, y) \subset S^{1}$ is $q_{n}$-small and $f^{s}(x), f^{s}(y) \notin B P(f)$ for all $0 \leqslant s<q_{n}$, then for any $k \in\left[0, q_{n}\right)$ Finzi's inequality

$$
e^{-v} \leqslant \frac{D f^{k}(x)}{D f^{k}(y)} \leqslant e^{v}
$$

holds, where $v$ is the total variation of $\log D f$ on $S^{1}$;

- for any $y_{0}$ with $y_{s}:=f^{s}\left(y_{0}\right) \notin B P(f)$ for all $0 \leqslant s<q_{n}$, the inequality

$$
e^{-v} \leqslant \prod_{s=0}^{q_{n}-1} D f\left(y_{s}\right) \leqslant e^{v}
$$

holds. This inequality is called Denjoy's inequality;

- for an arbitrary element $I^{(n)}$ of the dynamical partition $\xi_{n}\left(x_{0}\right)$

$$
\ell\left(I^{(n)}\right) \leqslant \operatorname{const} \lambda^{n}
$$

holds, where $\lambda=\left(1+e^{-v}\right)^{-\frac{1}{2}}<1$;

- the homeomorphism $f$ is topologically conjugate to the linear rotation $R_{\rho}$ i.e., there exists a homeomorphism $\varphi$ such that $\varphi \circ f=f_{\rho} \circ \varphi$;

- let $\mu_{f}$ be the unique invariant probability measure $\mu_{f}$ of $f$. Then (see $[14$, p. 71])

$$
\int_{S^{1}} \log D f(x) d \mu_{f}(x)=0 .
$$

\section{Herman's family of $\boldsymbol{P L}$-homeomorphisms with two break points}

In [14, Section 7 of Chapter VI] M. Herman introduced a family of $P L$-homeomorphisms with two break points, for which he studied their invariant measures and the regularity of the 


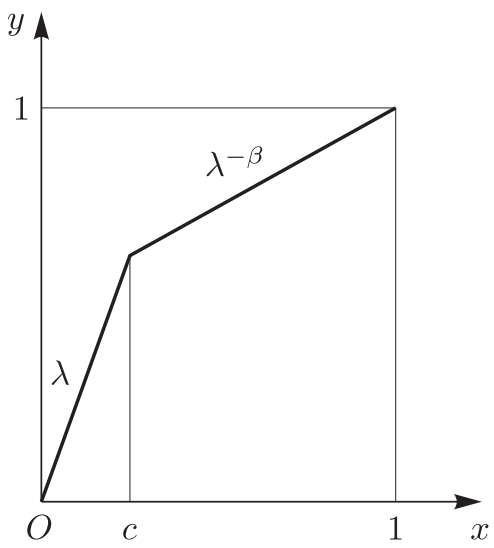

Fig. 1

maps conjugating them to linear rotations: given two real numbers $\lambda>1$ and $\beta>0$, he defines for $\widehat{x} \in[0,1]$ the piecewise linear map $H_{\beta, \lambda}:[0,1] \rightarrow[0,1]$ as

$$
H_{\beta, \lambda}(\widehat{x})= \begin{cases}\lambda \widehat{x}, & \text { if } 0 \leqslant \widehat{x} \leqslant c, \\ \lambda^{-\beta}(\widehat{x}-1)+1, & \text { if } c \leqslant \widehat{x} \leqslant 1,\end{cases}
$$

such that $\lambda c=\lambda^{-\beta}(c-1)+1$.

Then Herman considered for $0 \leqslant \theta \leqslant 1$ the one-parameter family of $P L$-maps $H_{\beta, \lambda, \theta}$ of the unit interval with

$$
H_{\beta, \lambda, \theta}(\widehat{x})=H_{\beta, \lambda}(\widehat{x})+\theta \quad \bmod 1,
$$

and the induced piecewise linear homeomorphisms of the circle

$$
h_{\beta, \lambda, \theta}(x)=H_{\beta, \lambda, \theta}(\widehat{x}) \bmod 1 .
$$

Obviously, $a_{0}=0$ and $c_{0}=c$ are break points of all these $h_{\beta, \lambda, \theta}$. Denote their rotation number for fixed $\lambda>1$ and $\beta>0$ by $\rho_{\theta}$. Continuity and monotonicity of $\rho_{\theta}$ as a function of $\theta$ imply that for an arbitrary irrational number $\alpha \in[0,1]$ there exists a unique $\theta=\theta(\alpha) \in[0,1]$ with $\rho_{\theta}=\alpha$. Herman then proved in [14]

Theorem 4. The following properties are equivalent:

(i) $h_{\beta, \lambda, \theta}$ is conjugate to the linear rotation $R_{\alpha}$ through an absolutely continuous homeomorphism;

(ii) $h_{\beta, \lambda, \theta}$ is conjugate to $R_{\alpha}$ through a Lipschitz homeomorphism;

(iii) $h_{\beta, \lambda, \theta}$ can be conjugated to $R_{\alpha}$ by a piecewise $C^{\infty}$ homeomorphism, which is not $P L$;

(iv) $\frac{\beta}{\beta+1} \in \mathbb{Z} \alpha \bmod 1$;

(v) the break points $a_{0}$ and $c_{0}$ belong to the same orbit under $h_{\beta, \lambda, \theta}$.

It can be easily checked (see [14]) that up to two points

$$
\frac{\log D h_{\beta, \lambda, \theta}(x)}{(1+\beta) \log \lambda}=\chi_{\left[a_{0}, c_{0}\right]}(x)-\frac{\beta}{1+\beta},
$$

where $\chi_{\left[a_{0}, c_{0}\right]}$ is the characteristic function of the interval $\left[a_{0}, c_{0}\right]$. Obviously,

$$
\sigma=\sigma\left(a_{0}\right)=\frac{D h_{-}(0)}{D h+(0)}=\lambda^{-1-\beta}
$$


for $h=h_{\beta, \lambda, \theta}$, and hence $\log \sigma=-(1+\beta) \log \lambda$. Then we can rewrite (3.2) as

$$
\frac{\log D h_{\beta, \lambda, \theta}(x)}{\log \sigma}=\frac{\beta}{1+\beta}-\chi_{\left[a_{0}, c_{0}\right]}(x),
$$

and hence also for any $n \geqslant 1$

$$
\frac{\log D h_{\beta, \lambda, \theta}^{n}(x)}{\log \sigma}=\frac{n \beta}{1+\beta}-\sum_{k=0}^{n-1} \chi_{\left[a_{0}, c_{0}\right]}\left(h_{\beta, \lambda, \theta}^{k}(x)\right) .
$$

Therefore, the following useful lemma holds for Herman's homeomorphism $h_{\beta, \lambda, \theta}$

Lemma 1. For every $n \geqslant 1$

$$
\frac{1}{\log \sigma} \log D h_{\beta, \lambda, \theta}^{n}(x)=n \frac{\beta}{1+\beta} \bmod 1 .
$$

Furthermore, one has

Lemma 2. Let $\mu_{\theta}$ be the invariant measure of Herman's map $h_{\beta, \lambda, \theta}$ with break points $a_{0}, c_{0}$ and an irrational rotation number $\rho_{\theta}$. Then

$$
\mu_{\theta}\left(\left[a_{0}, c_{0}\right]\right)=\frac{\beta}{1+\beta} .
$$

Proof. By Eq. (2.6) one has $\int_{S^{1}} \frac{\log D h_{\beta, \lambda, \theta}(x)}{\log \sigma} d \mu_{\theta}(x)=0$. Inserting for $\frac{\log D h_{\beta, \lambda, \theta}(x)}{\log \sigma}$ the right-hand side of (3.3), we get

$$
\int_{S^{1}}\left\{\frac{\beta}{1+\beta}-\chi_{\left[a_{0}, c_{0}\right]}(x)\right\} d \mu_{\theta}(x)=\frac{\beta}{1+\beta}-\mu_{\theta}\left(\left[a_{0}, c_{0}\right]\right)=0,
$$

and hence the lemma is proved.

REMARK 4. In (3.6) the right-hand side does not depend on the parameter $\theta$.

The uniform distribution of sequences is one of the classical problems of ergodic theory (see, for instance, [17]). Indeed, one has

Theorem 5 (see $[\mathbf{1 7}]$ ). For $[a, b] \subset \mathbb{R}$ let $u_{n}:[a, b] \rightarrow \mathbb{R}, n=1,2, \ldots$, be a sequence of continuously differentiable real-valued functions. Suppose, for arbitrary $m, n \in \mathbb{N}, n \neq m$, the function $D u_{n}(x)-D u_{m}(x)$ is monotone with respect to $x$ and that furthermore $\mid D u_{n}(x)-$ $-D u_{m}(x) \mid \geqslant K>0$ for some constant $K$ not depending on $x, m$ and $n$. Then the sequence $u_{n}(x), n=1,2, \ldots$ is uniformly distributed $\bmod 1$ for almost all $x$ in $[a, b]$.

Since $u_{n}(x)=q_{n} \cdot x$ fulfills the assumptions of Theorem 5 , it follows that the sequence $\frac{q_{n} \cdot \beta}{1+\beta} \bmod 1$ is uniformly distributed for Lebesgue almost all $\frac{\beta}{1+\beta}$. Clearly, this sequence is not uniformly distributed for all $\frac{\beta}{1+\beta}$, since for $\frac{\beta}{1+\beta}=m \rho_{f_{\beta, \lambda, \theta}} \bmod 1$ for some integer $m$, $\lim _{n \rightarrow \infty}\left\|\frac{q_{n} \cdot \beta}{1+\beta}\right\|=0$, where $\|x\|$ denotes the distance of $x$ to the nearest integer. In the case of rotation numbers of bounded type one has indeed the following result. 
Theorem 6 (see $[\mathbf{1 7}, \mathbf{1 8}])$. Let $\alpha$ be an irrational number of bounded type with partial quotients $\frac{p_{n}}{q_{n}}$. Then

$$
\lim _{n \rightarrow \infty}\left\|q_{n} x\right\|=0
$$

if and only if $x \in \mathbb{Z} \alpha \bmod 1$.

\section{On the location of break points}

In this section we recall four lemmas from [10] concerning the break points for an arbitrary $P$-homeomorphism $f$ with an irrational rotation number $\rho_{f}$ and two break points $a_{0}=a_{0}^{*}$ and $c_{0}=c_{0}^{*}$ not on the same orbit. They describe the locations of break points of the map $f^{q_{n}}$ and the values of the derivative $D f^{q_{n}}$ on $S^{1}$, where $\frac{p_{n}}{q_{n}}$ are the partial quotients of $\rho_{f}$. Obviously, the map $f^{q_{n}}$ has $2 q_{n}$ break points denoted by $B P\left(f^{q_{n}}\right):=B P\left(f^{q_{n}} ; a_{0}^{*}\right) \cup B P\left(f^{q_{n}} ; c_{0}^{*}\right)$ with $B P\left(f^{q_{n}} ; a_{0}^{*}\right):=\left\{a_{0}^{*}, a_{-1}^{*}, \ldots, a_{-q_{n}+1}^{*}\right\}$, respectively, $B P\left(f^{q_{n}} ; c_{0}^{*}\right):=\left\{c_{0}^{*}, c_{-1}^{*}, \ldots, c_{-q_{n}+1}^{*}\right\}$, where $a_{-i}^{*}=f^{-i}\left(a_{0}^{*}\right)$, respectively, $c_{-i}^{*}=f^{-i}\left(c_{0}^{*}\right), 0 \leqslant i \leqslant q_{n}-1$.

It is clear that the break points of the map $f^{q_{n}}$ define a partition $\mathcal{P}_{n}(f)$ of the circle $S^{1}$ into $2 q_{n}$ intervals with pairwise non-intersecting interior.

Let $\xi_{n}\left(a_{0}^{*}\right)$ be the $n$th dynamical partition determined by the break point $a_{0}=a_{0}^{*}$ with respect to the map $f$. Then one has for the second break point $c_{0}^{*}$ either $c_{0}^{*} \in I_{i_{0}}^{(n)}\left(a_{0}\right)$ for some $0 \leqslant i_{0}<q_{n-1}$, or $c_{0}^{*} \in I_{j_{0}}^{(n-1)}\left(a_{0}\right)=f^{j_{0}}\left(\left(a_{0}, a_{-q_{n}}\right]\right) \cup f^{j_{0}}\left(\left(a_{-q_{n}}, a_{q_{n-1}}\right)\right)$ for some $0 \leqslant j_{0}<q_{n}$, i.e., $c_{0}^{*} \in f^{j_{0}}\left(\left(a_{0}, a_{-q_{n}}\right]\right)$ or $c_{0}^{*} \in f^{j_{0}}\left(\left(a_{-q_{n}}, a_{q_{n-1}}\right)\right)$. The two last cases have to be treated separately. The following three lemmas describe the location of the break points of $f^{q_{n}}$ in intervals of different $n$ dynamical partitions (for the proofs see [10]).

Lemma 3 ([10]). Assume $c_{0}^{*} \in I_{i_{0}}^{(n)}\left(a_{0}^{*}\right)$ for some $i_{0}$ with $0 \leqslant i_{0}<q_{n-1}$. Then the break points $\left\{a_{-i}^{*}, c_{-i}^{*}, 0 \leqslant i \leqslant q_{n}-1\right\}$ of $f^{q_{n}}$ belong to the following elements of the dynamical partition $\xi_{n}\left(a_{0}^{*}\right)$ (see also Fig. 2):

- $a_{0}^{*} \in I_{0}^{(n)}\left(a_{0}^{*}\right)$;

- $c_{-i_{0}+s}^{*}=f^{s}\left(c_{-i_{0}}^{*}\right) \in I_{s}^{(n)}\left(a_{0}^{*}\right), 0 \leqslant s \leqslant i_{0}$;

- $a_{-q_{n}+s}^{*}=f^{s}\left(a_{-q_{n}}\right) \in f^{s}\left(\left(a_{0}^{*}, a_{-q_{n}}\right]\right) \subset I_{s}^{(n-1)}\left(a_{0}^{*}\right), \quad 1 \leqslant s \leqslant i_{0}$;

- $a_{-q_{n}+s}^{*}, c_{-q_{n}-i_{0}+s}^{*}=f^{s}\left(c_{-q_{n}-i_{0}}\right) \in f^{s}\left(\left(a_{0}^{*}, a_{-q_{n}}\right]\right) \subset I_{s}^{(n-1)}\left(a_{0}^{*}\right), i_{0}+1 \leqslant s \leqslant q_{n}-1$.

Lemma 4 ([10]). Assume $c_{0}^{*} \in f^{i_{0}}\left(\left(a_{0}^{*}, a_{-q_{n}}\right]\right)$ for some $0 \leqslant i_{0}<q_{n}$. Then the break points of $f^{q_{n}}$ belong to the following elements of the dynamical partition $\xi_{n}\left(c_{-i_{0}}^{*}\right)$ of the break point $c_{-i_{0}}^{*}$ (see Fig. 3):

- $c_{-i_{0}}^{*}, a_{0}^{*} \in I_{0}^{(n)}\left(c_{-i_{0}}^{*}\right)$

- $c_{-i_{0}+s}^{*}=f^{s}\left(c_{-i_{0}}^{*}\right), \quad a_{-q_{n}+s}^{*}=f^{s}\left(a_{-q_{n}}\right) \in f^{s}\left(\left[c_{-i_{0}}^{*}, a_{-q_{n}}\right]\right) \subset I_{s}^{(n-1)}\left(c_{-i_{0}}^{*}\right), \quad 1 \leqslant s \leqslant i_{0}$;

- $c_{-q_{n}-i_{0}+s}^{*}=f^{s}\left(c_{-q_{n}-i_{0}}^{*}\right), a_{-q_{n}+s}^{*}=f^{s}\left(a_{-q_{n}}\right) \in f^{s}\left(\left[c_{-i_{0}}, c_{-q_{n}}\right]\right) \subset I_{s}^{(n-1)}\left(c_{-i_{0}}^{*}\right)$, $i_{0}+1 \leqslant s \leqslant q_{n}-1$. 


\begin{tabular}{|c|c|c|c|c|c|}
\hline$a_{q_{n}}$ & $c_{-i_{0}}^{*}$ & $a_{0}^{*}$ & $c_{-i_{0}-q_{n}}$ & $a_{-q_{n}}$ & $a_{q_{n-1}}$ \\
\hline$a_{q_{n}+1}$ & $c_{-i_{0}+1}^{*}$ & $a_{1}$ & $c_{-i_{0}-q_{n}+1}$ & $a_{-q_{n}+1}^{*}$ & $a_{q_{n-1}+1}$ \\
\hline$a_{q_{n}+i_{0}}$ & $c_{0}^{*}$ & $a_{i_{0}}$ & $c_{-q_{n}}$ & $a_{-q_{n}+i_{0}}^{*}$ & $a_{q_{n-1}+i_{0}}$ \\
\hline$a_{q_{n}+i_{0}+1}$ & $c_{1}$ & $a_{i_{0}+1}$ & $c_{-q_{n}+1}^{*}$ & $a_{-q_{n}+i_{0}+1}^{*}$ & $a_{q_{n-1}+i_{0}+1}$ \\
\hline$a_{2 q_{n}-1}$ & $c_{q_{n}-i_{0}-1}$ & $a_{q_{n}-1}$ & $c_{-i_{0}-1}^{*}$ & $a_{-1}^{*}$ & $a_{q_{n-1}+q_{n}-1}$ \\
\hline
\end{tabular}

Fig. 2

\begin{tabular}{cccccc}
\hline$c_{-i_{0}+q_{n}}$ & $a_{0}^{*}$ & $c_{-i_{0}}^{*}$ & $a_{-q_{n}}$ & $c_{-i_{0}-q_{n}}$ & $c_{-i_{0}+q_{n-1}}$ \\
\hline$c_{-i_{0}+q_{n}+1}$ & $a_{1}$ & $c_{-i_{0}+1}^{*}$ & $a_{-q_{n}+1}^{*}$ & $c_{-i_{0}-q_{n}+1}$ & $c_{-i_{0}+q_{n-1}+1}$
\end{tabular}

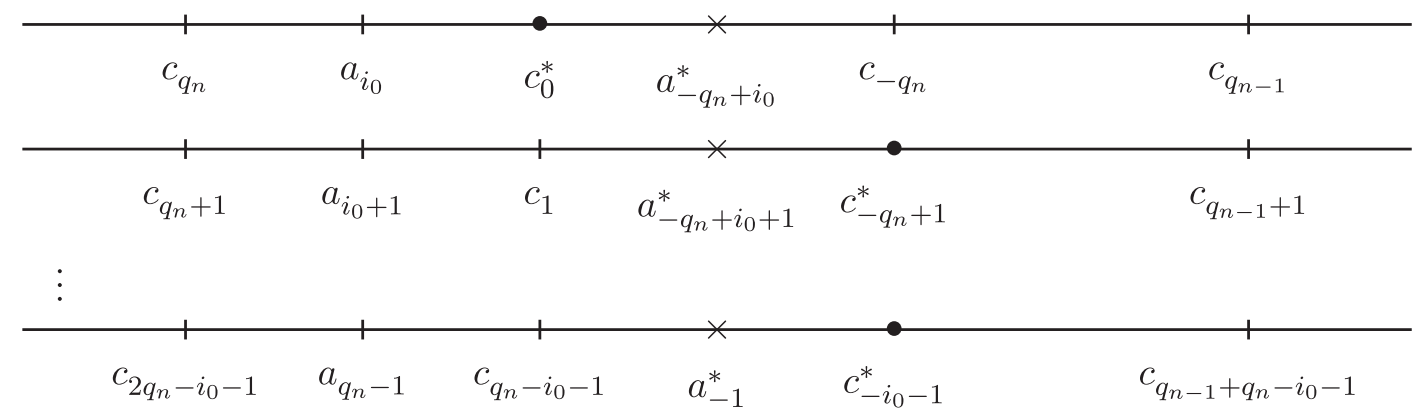

Fig. 3

Lemma 5 ([10]). If $c_{0}^{*} \in f^{i_{0}}\left(\left(a_{-q_{n}}, a_{q_{n-1}}\right]\right)$ for some $i_{0}$ with $0 \leqslant i_{0}<q_{n}$, the break points of $f^{q_{n}}$ are located in the following elements of the dynamical partition $\xi_{n}\left(a_{-q_{n}+1}^{*}\right)$ of the break point $a_{-q_{n}+1}^{*}$ (see also Fig. 4):

- $a_{-q_{n}+1+s}^{*} \in I_{s}^{(n-1)}\left(a_{-q_{n}+1}^{*}\right), \quad c_{-i_{0}+1+s}^{*} \in I_{s}^{(n-1)}\left(a_{-q_{n}+1}^{*}\right), \quad 0 \leqslant s \leqslant i_{0}-1$;

- $a_{-q_{n}+i_{0}+1+s}^{*} \in I_{i_{0}+s}^{(n-1)}\left(a_{-q_{n}+1}^{*}\right), \quad c_{-q_{n}+1+s}^{*} \in I_{i_{0}+s}^{(n-1)}\left(a_{-q_{n}+1}^{*}\right), \quad 0 \leqslant s \leqslant q_{n}-i_{0}-1$. 


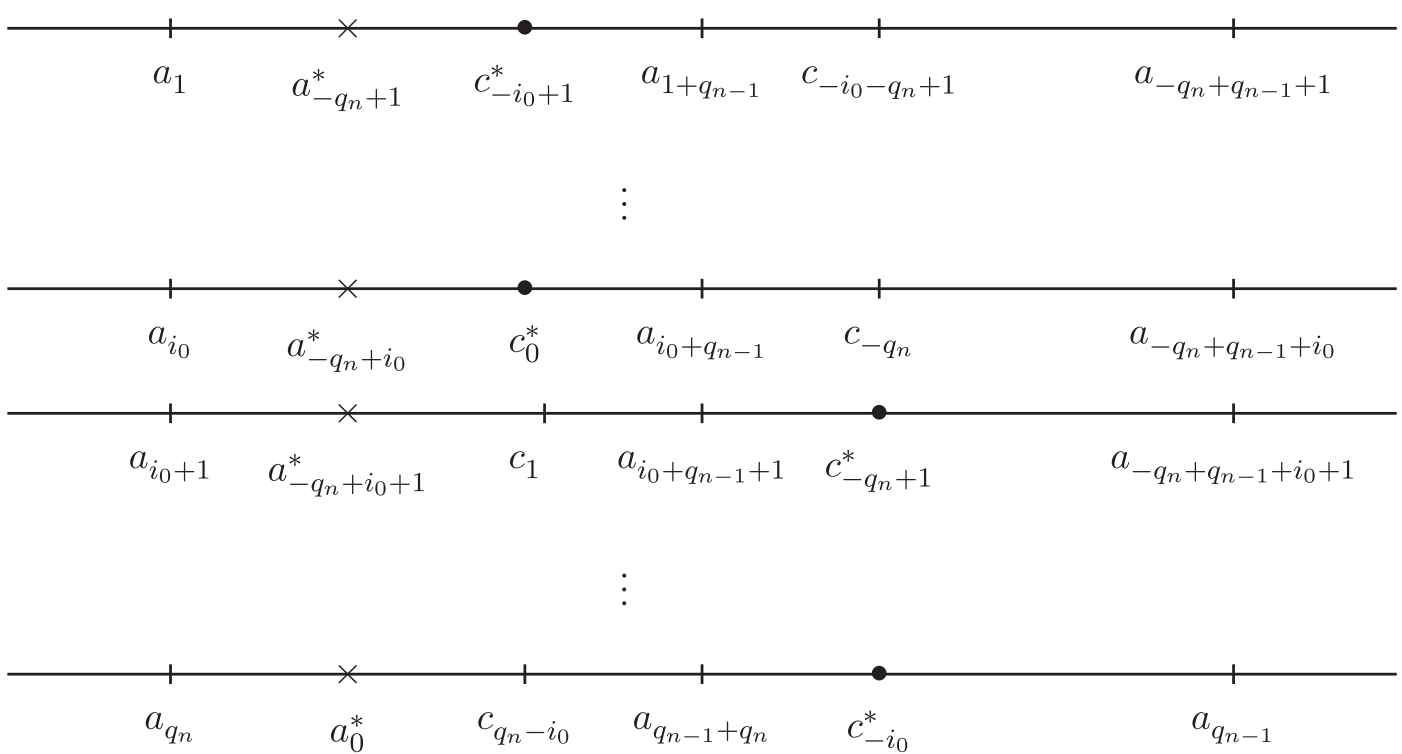

Fig. 4

Next we recall the case of a $P$-homeomorphism $f$ with an irrational rotation number $\rho_{f}$ and two break points $a_{0}^{*}:=a_{0}, a_{i_{0}}^{*}:=f^{i_{0}}\left(a_{0}\right), i_{0}>0$, on the same orbit. Put $n_{i_{0}}:=\min \left\{n: q_{n} \geqslant i_{0}\right\}$. Assume that $n>n_{i_{0}}$. If the total jump ratio is $\sigma_{f}=1$, the map $f^{q_{n}}$ has $2 i_{0}$ break points

$$
a_{-q_{n}+1}^{*}:=a_{-q_{n}+1}, \quad a_{-q_{n}+2}^{*}:=a_{-q_{n}+2}, \quad \ldots, \quad a_{-q_{n}+i_{0}}^{*}:=a_{-q_{n}+i_{0}}
$$

and

$$
a_{1}^{*}:=a_{1}, a_{2}^{*}:=a_{2}, \ldots, a_{i_{0}}^{*}:=a_{i_{0}} .
$$

If $\sigma_{f} \neq 1$, the map $f^{q_{n}}$ has $q_{n}+i_{0}$ break points

$$
a_{-q_{n}+1}^{*}:=a_{-q_{n}+1}, \quad a_{-q_{n}+2}^{*}:=a_{-q_{n}+2}, \ldots, a_{0}^{*}:=a_{0}, \ldots, a_{i_{0}}^{*}:=a_{i_{0}} .
$$

Then one has

Lemma 6 ([10]). Assume $f$ is a P-homeomorphism with an irrational rotation number $\rho_{f}$ and two break points $a_{0}^{*}:=a_{0}, a_{i_{0}}^{*}:=f^{i_{0}}\left(a_{0}\right), i_{0}>0$, on the same orbit. Choose $n>n_{i_{0}}$.

1) If $\sigma_{f}=1$, then one finds for the break points $a_{-q_{n}+s+1}^{*}, a_{s+1}^{*}$ of $f^{q_{n}}$

- $a_{-q_{n}+s+1}^{*}, a_{s+1}^{*} \in f^{s}\left(\left[a_{1}^{*}, a_{-q_{n}+1}^{*}\right]\right) \subset I_{s+1}^{(n-1)}\left(a_{0}^{*}\right) \in \xi_{n}\left(a_{0}^{*}\right), 0 \leqslant s \leqslant i_{0}-1$;

(see Fig. 5);

2) if $\sigma_{f} \neq 1$, we have

- $a_{0}^{*} \subset I_{0}^{(n-1)}\left(a_{0}^{*}\right)$;

- $a_{-q_{n}+1+s}^{*}, a_{1+s}^{*} \in f^{s}\left(\left[a_{i_{0}+1}, a_{-q_{n}+i_{0}+1}^{*}\right]\right) \subset I_{i_{0}+1+s}^{(n-1)}\left(a_{0}^{*}\right), \quad 0 \leqslant s \leqslant q_{n}-i_{0}-2$.

- $a_{s+1}^{*} \in f^{s}\left(\left[a_{1}^{*}, a_{-q_{n}+1}^{*}\right]\right) \subset I_{1+s}^{(n-1)}\left(a_{0}^{*}\right), \quad i_{0} \leqslant s \leqslant q_{n}-i_{0}-1$. 


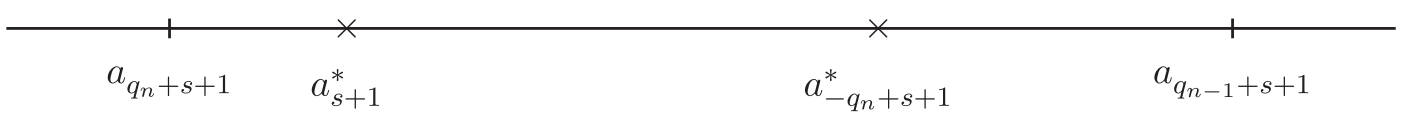

Fig. 5

Lemmas 3 to 6 show the location of the break points of $f^{q_{n}}$ on elements of different $n$th dynamical partitions determined by the map $f$, respectively, their order along the circle. Indeed, these lemmas hold true also for any pure rotation $R_{\rho}$ with $\rho$ irrational and any two points $a_{0}, c_{0} \in S^{1}$, whose preimages under $R_{\rho}^{q_{n}}$ correspond to the break points of the $P$-homeomorphism $f^{q_{n}}$.

Lemmas 3-5 imply the following corollary.

Corollary 1. Let $h$ be a PL-homeomorphism with two break points $a_{0}$ and $c_{0}$. Then each interval of the partition $\xi_{n}^{*}$ contains exactly one $z \in B P\left(h^{q_{n}} ; a_{0}\right)$ and one $z \in B P\left(h^{q_{n}} ; c_{0}\right)$.

\section{Denjoy equality for piecewise linear circle homeomorphisms with two break points}

In this section we recall the explicit expressions derived in [10] for the derivatives $D h^{q_{n}}$ of the piecewise linear homeomorphism $h$ with two break points $a_{0}, c_{0}$ in terms of the jump ratio $\sigma_{h}\left(a^{(0)}\right)$ and the $\mu_{h}$-measures of certain intervals of the partition $\mathcal{P}_{n}(h)$ of $S^{1}$ determined by the break points of $h^{q_{n}}$.

They follow from Lemmas 3-5 applied to a $P L$-circle homeomorphism $h$ with an irrational rotation number $\rho_{h}$ and two break points $a_{0}^{*}=a_{0}=0, c_{0}^{*}=c_{0}$ not on the same orbit and with the total jump ratio $\sigma_{h}=1$.

In the case of Lemma 3 and Lemma 5 the break points $P B\left(h^{q_{n}} ; a_{0}^{*}\right)$ of $h^{q_{n}}$, associated with $a_{0}^{*}=0$, and $P B\left(h^{q_{n}} ; c_{0}^{*}\right)$, associated with $c_{0}^{*}$, alternate in their order along the circle $S^{1}$.

Let $n$ be odd. Obviously, the break points of $h^{q_{n}}$ define a system of disjoint subintervals of the circle, given in the case of the assumption in Lemma 3 by (see Fig. 2)

$$
\left[c_{-i_{0}+s}^{*}, a_{-q_{n}+s}^{*}\right], \quad 1 \leqslant s \leqslant i_{0},
$$

respectively,

$$
\left[c_{-i_{0}-q_{n}+s}^{*}, a_{-q_{n}+s}^{*}\right], \quad i_{0}+1 \leqslant s \leqslant q_{n} .
$$

We combine these subintervals to the subsets

$$
A_{n}\left(i_{0}\right):=\bigcup_{s=1}^{i_{0}}\left[c_{-i_{0}+s}^{*}, a_{-q_{n}+s}^{*}\right], \quad B_{n}\left(i_{0}\right):=\bigcup_{s=i_{0}+1}^{q_{n}}\left[c_{-i_{0}-q_{n}+s}^{*}, a_{-q_{n}+s}^{*}\right] .
$$

In the case of the assumption in Lemma 5 the subintervals are given by (see Fig. 4)

$$
\left[a_{-q_{n}+s}^{*}, c_{-i_{0}+s}^{*}\right], \quad 1 \leqslant s \leqslant i_{0},
$$

respectively,

$$
\left[a_{-q_{n}+s}^{*}, c_{-i_{0}-q_{n}+s}^{*}\right], \quad i_{0}+1 \leqslant s \leqslant q_{n},
$$


which we combine to the subsets

$$
A_{n}\left(i_{0}\right):=\bigcup_{s=1}^{i_{0}}\left[a_{-q_{n}+s}^{*}, c_{-i_{0}+s}^{*}\right], \quad B_{n}\left(i_{0}\right):=\bigcup_{s=i_{0}+1}^{q_{n}}\left[a_{-q_{n}+s}^{*}, c_{-i_{0}-q_{n}+s}^{*}\right] .
$$

For $n$ even, the orientation of the above intervals has to be reversed. Therefore, in the case of Lemma 3 we have the following system of disjoint intervals:

$$
\left[a_{-q_{n}+s}^{*}, c_{-i_{0}+s}^{*}\right], \quad 1 \leqslant s \leqslant i_{0},
$$

respectively,

$$
\left[a_{-q_{n}+s}^{*}, c_{-i_{0}-q_{n}+s}^{*}\right], \quad i_{0}+1 \leqslant s \leqslant q_{n} .
$$

In the case of Lemma 5 one finds

$$
\left[c_{-i_{0}+s}^{*}, a_{-q_{n}+s}^{*}\right], \quad 1 \leqslant s \leqslant i_{0},
$$

respectively,

$$
\left[c_{-i_{0}-q_{n}+s}^{*}, a_{-q_{n}+s}^{*}\right], \quad i_{0}+1 \leqslant s \leqslant q_{n} .
$$

In the case of Lemma 3 and $n$ even, respectively, in the case of Lemma 5 and $n$ odd, the subsets $A_{n}\left(i_{0}\right)$ and $B_{n}\left(i_{0}\right)$ can be defined as before. The above constructions show that the boundaries of every interval in the subsets $A_{n}\left(i_{0}\right)$ and $B_{n}\left(i_{0}\right)$ consist of break points from $P B\left(h^{q_{n}} ; a_{0}^{*}\right)$, respectively, $P B\left(h^{q_{n}} ; c_{0}^{*}\right)$. In the following we abbreviate the jump ratio of $h$ at the break point $a_{0}^{*}$ by

$$
\sigma:=\sigma_{h}\left(a_{0}^{*}\right)=\frac{D h_{-}(0)}{D h_{+}(0)} .
$$

Next, we recall the first result on the values of $\left(D h^{q_{n}}(x)\right)$ from [10].

Theorem 7. Let $h$ be a PL-circle homeomorphism with an irrational rotation number $\rho_{h}$ and two break points $a_{0}^{*}=0$ and $c_{0}^{*}$, whose total jump ratio is $\sigma_{h}=1$, and which lie on different orbits. Assume $c_{0}^{*}$ fulfills the assumptions of Lemma 3 with $0 \leqslant i_{0}<q_{n-1}$, respectively, Lemma 5 for some $i_{0}$ with $0 \leqslant i_{0}<q_{n}$. Then in the case of Lemma 3

$$
\left(D h^{q_{n}}(x)\right)^{(-1)^{n}}= \begin{cases}\sigma^{\mu_{h}\left(A_{n}\left(i_{0}\right) \cup B_{n}\left(i_{0}\right)\right)-1}, & \text { if } x \in A_{n}\left(i_{0}\right) \cup B_{n}\left(i_{0}\right), \\ \sigma^{\mu_{h}\left(A_{n}\left(i_{0}\right) \cup B_{n}\left(i_{0}\right)\right)}, & \text { if } x \in S^{1} \backslash\left(A_{n}\left(i_{0}\right) \cup B_{n}\left(i_{0}\right)\right) ;\end{cases}
$$

respectively, in the case of Lemma 5 ,

$$
\left(D h^{q_{n}}(x)\right)^{(-1)^{n+1}}= \begin{cases}\sigma^{\mu_{h}\left(A_{n}\left(i_{0}\right) \cup B_{n}\left(i_{0}\right)\right)-1}, & \text { if } x \in A_{n}\left(i_{0}\right) \cup B_{n}\left(i_{0}\right), \\ \sigma^{\mu_{h}\left(A_{n}\left(i_{0}\right) \cup B_{n}\left(i_{0}\right)\right)}, & \text { if } x \in S^{1} \backslash\left(A_{n}\left(i_{0}\right) \cup B_{n}\left(i_{0}\right)\right) .\end{cases}
$$

Theorem 7 shows that $D h^{q_{n}}$ is constant on every element of the partition $\mathcal{P}_{n}(h)$ and takes only two values under the assumptions of Lemmas 3 and 5 . Moreover, the values of $D h^{q_{n}}$ are determined by the jump ratio $\sigma=\sigma_{h}\left(a_{0}^{*}\right)$ and the $\mu_{h}$-measure of $A_{n}\left(i_{0}\right) \cup B_{n}\left(i_{0}\right)$.

In the case of the assumption on $c_{0}^{\star}$ in Lemma 4 we can define again a system of disjoint subintervals determined by the elements in $\mathcal{P}_{n}(h)$. Let $n$ be odd. Then these subintervals are as follows (see Fig. 3):

$$
\left[c_{-i_{0}+s}^{*}, a_{-q_{n}+s}^{*}\right], \quad 1 \leqslant s \leqslant i_{0},
$$


respectively,

$$
\left[a_{-q_{n}+s}^{*}, c_{-i_{0}-q_{n}+s}^{*}\right], \quad i_{0}+1 \leqslant s \leqslant q_{n} .
$$

For $n$ even the orientation of the above intervals has to be reversed. To determine in the case of Lemma 4 the values of $D f^{q_{n}}$, we define

$$
A_{n}\left(i_{0}\right):=\bigcup_{s=1}^{i_{0}}\left[c_{-i_{0}+s}^{*}, a_{-q_{n}+s}^{*}\right], \quad B_{n}\left(i_{0}\right):=\bigcup_{s=i_{0}+1}^{q_{n}}\left[a_{-q_{n}+s}^{*}, c_{-i_{0}-q_{n}+s}^{*}\right] .
$$

Then the following theorem holds.

Theorem 8 ([10]). Let $h$ be a PL-circle homeomorphism with two break points $a_{0}^{*}$ and $c_{0}^{*}$ with $\sigma_{h}=1$, which lie on different orbits. Assume $c_{0}^{*}$ fulfills the assumption of Lemma 4 for some $i_{0}$ with $0 \leqslant i_{0}<q_{n}$. Then for all $n \geqslant 1$

$$
\left(D h^{q_{n}}(x)\right)^{(-1)^{n}}= \begin{cases}\sigma^{\mu_{h}\left(A_{n}\left(i_{0}\right)\right)-\mu_{h}\left(B_{n}\left(i_{0}\right)\right)-1} & \text { if } x \in A_{n}\left(i_{0}\right), \\ \sigma^{\mu_{h}\left(A_{n}\left(i_{0}\right)\right)-\mu_{h}\left(B_{n}\left(i_{0}\right)\right)+1} & \text { if } x \in B_{n}\left(i_{0}\right), \\ \sigma^{\mu_{h}\left(A_{n}\left(i_{0}\right)\right)-\mu_{h}\left(B_{n}\left(i_{0}\right)\right)} & \text { if } x \notin A_{n}\left(i_{0}\right) \cup B_{n}\left(i_{0}\right) .\end{cases}
$$

It remains to discuss the case of a $P L$-homeomorphism $h$ with an irrational rotation number $\rho_{h}$ and two break points $a_{0}^{*}=0$ and $a_{i_{0}}^{*}=h^{i_{0}}\left(a_{0}^{*}\right), i_{0}>0$, on the same orbit. In this case the break points of $h^{q_{n}}$ alternate in their order along the circle $S^{1}$. Denote by $U_{n}\left(a_{s}^{*}\right), 1 \leqslant s \leqslant i_{0}$, the closed intervals with endpoints $a_{s}^{*}$ and $a_{-q_{n}+s}^{*}$. Obviously, these subintervals are disjoint. Lemma 6 implies that $U_{n}\left(a_{s}^{*}\right) \subset I_{s}^{(n-1)}\left(a_{0}^{*}\right), 1 \leqslant s \leqslant i_{0}$. Next, we define for every $n \geqslant 1$

$$
U_{n}=\bigcup_{s=1}^{i_{0}} U_{n}\left(a_{s}^{*}\right)
$$

Then one has

Theorem 9 ([10]). Let $h$ be a PL-circle homeomorphism with two break points $a_{0}^{*}=0$ and $a_{i_{0}}^{*}=h^{i_{0}}\left(a_{0}\right), i_{0}>0$, with $\sigma_{h}=1$, which lie on the same orbit. Put $n_{i_{0}}:=\min \left\{n: q_{n} \geqslant i_{0}\right\}$. For $n>n_{i_{0}}$ one finds

$$
\left(D h^{q_{n}}(x)\right)^{(-1)^{n+1}}= \begin{cases}\sigma^{\mu_{h}\left(U_{n}\right)} & \text { if } x \in U_{n}, \\ \sigma^{\mu_{h}\left(U_{n}\right)-1} & \text { if } x \in S^{1} \backslash U_{n} .\end{cases}
$$

Theorems 7 and 8 lead to the following

Theorem 10. Let $h$ be a PL-circle homeomorphism with an irrational rotation number $\rho_{h}$ and two break points $a_{0}^{*}=0$ and $c_{0}^{*}$, which lie on different orbits. Assume $c_{0}^{*}$ fulfills the assumptions in Lemma 3 for some $i_{0}$ with $0 \leqslant i_{0}<q_{n-1}$, respectively, in Lemma 4 or Lemma 5 for some $i_{0}$ with $0 \leqslant i_{0}<q_{n}$. Then in the case of Lemma 3 or Lemma 4

$$
\frac{1}{\log \sigma} \log D h^{q_{n}}(x)=(-1)^{n}\left(\mu_{h}\left(A_{n}\left(i_{0}\right)\right)+\mu_{h}\left(B_{n}\left(i_{0}\right)\right)\right)=q_{n} \cdot \frac{\beta}{1+\beta} \bmod 1,
$$

respectively, in the case of Lemma 5 ,

$$
\frac{1}{\log \sigma} \log D h^{q_{n}}(x)=(-1)^{n+1}\left(\mu_{h}\left(B_{n}\left(i_{0}\right)\right)-\mu_{h}\left(A_{n}\left(i_{0}\right)\right)\right)=q_{n} \cdot \frac{\beta}{1+\beta} \bmod 1 .
$$




\section{Proof of Theorem 3}

Let $f \in C^{2+\epsilon}\left(S^{1} \backslash\left\{b_{1}, b_{2}\right\}\right)$ be a $P$-homeomorphism of the circle with an irrational rotation number $\rho_{f}$ and two break points $b_{1}$ and $b_{2}$ not on the same orbit, whose total jump ratio is $\sigma_{f}=\sigma_{f}\left(b_{1}\right) \cdot \sigma_{f}\left(b_{2}\right)=1$. Denote by $\mu_{f}$ its unique invariant probability measure. Define the parameters $\beta$ and $\lambda$ through

$$
\frac{\beta}{1+\beta}:=\mu_{f}\left(\left[b_{1}, b_{2}\right]\right), \quad \lambda^{-1-\beta}:=\sigma_{f}\left(b_{1}\right) .
$$

Let $h=h_{\beta, \lambda, \theta}$ be Herman's $P L$-homeomorphism of $S^{1}$ with break points $a_{0}=0$ and $c_{0}=c$ such that $\lambda c=\lambda^{-\beta}(c-1)+1$. Since the rotation number $\rho_{f}$ is irrational, we can find a unique $\bar{\theta}$ such that the rotation number $\rho_{\bar{\theta}}$ of $h_{\bar{\theta}}=h_{\beta, \lambda, \bar{\theta}}$ coincides with $\rho_{f}$. Denote by $\mu_{\bar{\theta}}$ the invariant measure of $h_{\bar{\theta}}$. By Lemma $2 \mu_{\bar{\theta}}\left(\left[a_{0}, c_{0}\right]\right)=\frac{\beta}{1+\beta}$. Since $\rho_{f}=\rho_{\bar{\theta}}$, the homeomorphisms $f$ and $h_{\bar{\theta}}$ are topologically conjugate via some homeomorphism $\varphi$. We can choose $\varphi$ such that $a_{0}=\varphi\left(b_{1}\right)$ and $c_{0}=\varphi\left(b_{2}\right)$, because $\mu_{f}\left(\left[b_{1}, b_{2}\right]\right)=\mu_{\bar{\theta}}\left(\left[a_{0}, c_{0}\right]\right)$. Then one has also $\left(\varphi^{*} \mu_{\bar{\theta}}\right)\left(\left[b_{1}, b_{2}\right]\right)=\mu_{f}\left(\left[b_{1}, b_{2}\right]\right)=$ $=\mu_{\bar{\theta}}\left(\left[a_{0}, c_{0}\right]\right)$, since the invariant probability measure of $f$ is unique. Hence, we have proved the following fact, which will play a key role in our proof of the main theorem.

Theorem 11. Consider the P-homeomorphism $f: S^{1} \rightarrow S^{1}$ with an irrational rotation number $\rho_{f}$ and two break points $b_{1}, b_{2}$ with jump ratios $\sigma:=\sigma_{f}\left(b_{1}\right)$ and $\sigma_{f}\left(b_{2}\right)=\sigma^{-1}$. Let $h_{\bar{\theta}}:=h_{\beta, \lambda, \bar{\theta}}$ be Herman's PL-homeomorphism with two break points $a_{0}=0, c_{0}$, parameters $\beta, \lambda$ as defined by (6.1) and an irrational rotation number $\rho_{\bar{\theta}}=\rho_{f}$. Then

(I) $\sigma_{\bar{\theta}}\left(a_{0}\right)=\sigma$ and $\mu_{\bar{\theta}}\left(\left[a_{0}, c_{0}\right]\right)=\mu_{f}\left(\left[b_{1}, b_{2}\right]\right)$;

(II) the maps $f$ and $h_{\bar{\theta}}$ are topologically conjugate by some circle homeomorphism $\varphi$ with $\varphi\left(b_{1}\right)=a_{0}$ and $\varphi\left(b_{2}\right)=c_{0}$.

Since the rotation number $\rho_{f}$ is irrational, the invariant probability measure $\mu_{f}$ has no discrete ergodic component. Indeed, one knows that every such $P$-homeomorphism is ergodic also w.r.t. Lebesgue measure $l$ (see [14]). Suppose $\mu_{f}$ has an absolutely continuous component $\mu_{f}^{a . c}$ with support $A$ and $\mu_{f}^{a . c}(A)>0$. Then also $l(A)>0$. If $p(x)$ is the density of $\mu_{f}^{a . c}$, then on $A$ obviously $p(x) \geqslant 0$ and on $S^{1} \backslash A$ one has $p(x)=0$. Since $p(x)$ satisfies the functional equation $p(f(x))=\frac{1}{D f(x)} p(x), x \in S^{1}$ and $D f(x) \geqslant$ const $>0$, the subset $A_{+}=\{x: p(x)>0\}$ is $f$-invariant. Ergodicity of $f$ with respect to Lebesgue measure $l$ implies that either $\mu_{f}^{a . c}(A)=1$ or $\mu_{f}^{a . c}(A)=0$. Hence, the invariant measure $\mu_{f}$ is either pure absolutely continuous or pure singular on $S^{1}$.

The idea for the proof of Theorem 3 is to construct for the homeomorphism $f$ a sequence of measurable subsets $G_{n_{m}} \subset S^{1}$ such that $l\left(G_{n_{m}}\right)>$ const $>0$ and

$$
\liminf _{m \rightarrow \infty} l\left\{x \in G_{n_{m}:}\left|D f^{q_{n_{m}}}(x)-1\right|>\delta\right\}>0
$$

for some $\delta>0$. On the other hand, one knows that under the assumption of absolute continuity of the invariant measure $\mu_{f}$ w.r.t. Lebesgue measure $l, D f^{q_{n}}(x)$ tends, as $n \rightarrow \infty$, to 1 in probability with respect to the probability measure $l$.

Hence, let us start with the following proposition shown in [14]. 
Proposition 1. Let $f$ be a P-homeomorphism of the circle with an irrational rotation number $\rho_{f}$. If its invariant probability measure $\mu_{f}$ is equivalent to Lebesgue measure $l$, then for all $\delta>0$ the sequence $l\left(\left\{x:\left|D f^{q_{n}}(x)-1\right|>\delta\right\}\right)$ tends to zero as $n \rightarrow \infty$.

Important for the construction of the above mentioned sets $G_{n_{m}}$ will be

Lemma 7. For arbitrary $\delta \in(0,1)$ and $n \geqslant 1$ consider three points $z_{1}, z_{2}, z_{3} \in S^{1}$, $z_{2} \in B P\left(f^{q_{n}}\right)$, with $z_{1} \prec z_{2} \prec z_{3} \prec z_{1}$ such that the intervals $\left[z_{1}, z_{2}\right]$ and $\left[z_{2}, z_{3}\right]$ are $q_{n}$-small. Assume the P-homeomorphism $f^{q_{n}} \in C^{2+\varepsilon}\left(S^{1} \backslash\left\{z_{2}\right\}\right)$ has a jump ratio $\sigma_{f^{q_{n}}}\left(z_{2}\right)=\Lambda$ at the break point $z_{2}$. For $v$ the total variation of $\log D f$ on $S^{1}$ and $t_{l} \in\left(z_{1}, z_{2}\right)$ and $t_{r} \in\left(z_{2}, z_{3}\right)$ with

$$
\frac{l\left(\left[t_{l}, z_{2}\right]\right)}{l\left(\left[z_{1}, z_{2}\right]\right)}=\frac{l\left(\left[t_{r}, z_{3}\right]\right)}{l\left(\left[z_{2}, z_{3}\right]\right)}=\delta
$$

one has either

$$
\log D f^{q_{n}}(x) \leqslant-\frac{\log \Lambda}{2}+K e^{v} \delta
$$

for all $x \in\left[t_{l}, z_{2}\right)$, or

$$
\log D f^{q_{n}}(y) \geqslant \frac{\log \Lambda}{2}-K e^{v} \delta
$$

for all $y \in\left(z_{2}, t_{r}\right]$, when $\Lambda>1$.

In the case $\Lambda<1$ one has either

$$
\log D f^{q_{n}}(x) \geqslant-\frac{\log \Lambda}{2}-K e^{v} \delta,
$$

for all $x \in\left[t_{l}, z_{2}\right)$, or

$$
\log D f^{q_{n}}(y) \leqslant \frac{\log \Lambda}{2}+K e^{v} \delta
$$

for all $y \in\left(z_{2}, t_{r}\right]$.

Proof. Assume $\log \Lambda=\log \frac{D f_{-}^{q_{n}}\left(z_{2}\right)}{D f_{+}^{q_{n}}\left(z_{2}\right)}>0$, the case $\log \Lambda<0$ can be treated analogously. Then $\log D f_{-}^{q_{n}}\left(z_{2}\right)=\log \Lambda+\log D f_{+}^{q_{n}}\left(z_{2}\right)$, and hence,

$$
\log D f_{+}^{q_{n}}\left(z_{2}\right) \leqslant-\frac{\log \Lambda}{2} \text { if and only if } \log D f_{-}^{q_{n}}\left(z_{2}\right) \leqslant \frac{\log \Lambda}{2},
$$

respectively,

$$
\log D f_{+}^{q_{n}}\left(z_{2}\right) \geqslant-\frac{\log \Lambda}{2} \text { if and only if } \log D f_{-}^{q_{n}}\left(z_{2}\right) \geqslant \frac{\log \Lambda}{2}
$$

Hence, either

$$
\log D f_{+}^{q_{n}}\left(z_{2}\right) \leqslant-\frac{\log \Lambda}{2}
$$

or

$$
\log D f_{-}^{q_{n}}\left(z_{2}\right) \geqslant \frac{\log \Lambda}{2}
$$


Then for arbitrary $x \in\left[t_{l}, z_{2}\right)$ one finds

$$
\begin{aligned}
\left|\log \frac{D f_{-}^{q_{n}}\left(z_{2}\right)}{D f^{q_{n}}(x)}\right| & \leqslant \sum_{j=0}^{q_{n}-1}\left|\log D f_{-}\left(f^{j}\left(z_{2}\right)\right)-\log D f\left(f^{j}(x)\right)\right| \leqslant K \sum_{j=0}^{q_{n}-1} l\left(\left[f^{j}(x), f^{j}\left(z_{2}\right)\right]\right) \leqslant \\
& \leqslant K \sum_{j=0}^{q_{n}-1} l\left(\left[f^{j}\left(t_{l}\right), f^{j}\left(z_{2}\right)\right]\right) \leqslant K \sum_{j=0}^{q_{n}-1} \frac{l\left(\left[f^{j}\left(t_{l}\right), f^{j}\left(z_{2}\right)\right]\right)}{l\left(\left[f^{j}\left(z_{1}\right), f^{j}\left(z_{2}\right)\right]\right)} l\left(\left[f^{j}\left(z_{1}\right), f^{j}\left(z_{2}\right)\right]\right)= \\
& =K \sum_{j=0}^{q_{n}-1} \frac{D f^{j}(\zeta)}{D f^{j}(\vartheta)} \frac{l\left(\left[t_{l}, z_{2}\right)\right)}{l\left(\left[z_{1}, z_{2}\right)\right)} l\left(\left[f^{j}\left(z_{1}\right), f^{j}\left(z_{2}\right)\right]\right)
\end{aligned}
$$

for certain $\zeta \in\left[t_{l}, z_{2}\right), \vartheta \in\left[z_{1}, z_{2}\right)$ and an universal constant $K>0$ depending only on $f$. According to inequality (2.3),

$$
e^{-v} \leqslant \frac{D f^{j}(\zeta)}{D f^{j}(\vartheta)} \leqslant e^{v}
$$

and therefore

$$
K \sum_{j=0}^{q_{n}-1} \frac{D f^{j}(\zeta)}{D f^{j}(\vartheta)} \frac{l\left(\left[t_{l}, z_{2}\right)\right)}{l\left(\left[z_{1}, z_{2}\right)\right)} l\left(\left[f^{j}\left(z_{1}\right), f^{j}\left(z_{2}\right)\right]\right) \leqslant K e^{v} \delta,
$$

since $\frac{l\left(\left[t_{l}, z_{2}\right)\right)}{l\left(\left[z_{1}, z_{2}\right)\right)}=\delta$. We have also used the fact that the interval $\left[z_{1}, z_{2}\right]$ is $q_{n}$-small and hence the intervals $\left[f^{j}\left(z_{1}\right), f^{j}\left(z_{2}\right)\right], 0 \leqslant j \leqslant q_{n}-1$ are disjoint. This leads finally to the bound

$$
\left|\log \frac{D f_{-}^{q_{n}}\left(z_{2}\right)}{D f^{q_{n}}(x)}\right| \leqslant K e^{v} \delta
$$

In the same way it can be shown that

$$
\left|\log \frac{D f_{+}^{q_{n}}\left(z_{2}\right)}{D f^{q_{n}}(y)}\right| \leqslant K e^{v} \delta
$$

for all $y \in\left[t_{r}, z_{2}\right)$. Inserting the bounds (6.9)-(6.12), we get the bounds (6.3)-(6.6) in Lemma 7 .

An important role in the proof of Theorem 3 is played by certain neighborhoods of the break points of the $P$-homeomorphisms $f^{q_{n}}$, which we define next. For this, recall the partition $\mathcal{P}_{n}(f)$ of the circle defined by the breakpoints $B P\left(f^{q_{n}}\right)$ of the map $f^{q_{n}}$ in Section 4. For $z \in B P\left(f^{q_{n}}\right)$ we denote by $V_{n}^{l}(z)$, respectively, $V_{n}^{r}(z)$ the interval in $\mathcal{P}_{n}(f)$, whose right, respectively, left boundary point is the break point $z$. Given some $\delta \in(0,1)$, using Lemma 7 we can then construct left and right subintervals $V_{n}^{l}(z ; \delta) \subset V_{n}^{l}(z)$, respectively, $V_{n}^{r}(z ; \delta) \subset V_{n}^{r}(z)$, both with the break point $z$ as a boundary point, such that

$$
\frac{l\left(V_{n}^{l}(z ; \delta)\right)}{l\left(V_{n}^{l}(z)\right)}=\delta, \quad \frac{l\left(V_{n}^{r}(z ; \delta)\right)}{l\left(V_{n}^{r}(z)\right)}=\delta .
$$

Definition 3. The subintervals $V_{n}^{l}(z ; \delta), V_{n}^{r}(z ; \delta)$, respectively, the interval $V_{n}(z ; \delta)=$ $=V_{n}^{l}(z ; \delta) \cup V_{n}^{r}(z ; \delta)$ are called the left normalized $\delta$-neighborhood, the right normalized $\boldsymbol{\delta}$-neighborhood, respectively, the normalized $\boldsymbol{\delta}$-neighborhood of the break point $z$. 
Assume now there exists for $\rho=\rho_{f}=\rho_{\bar{\theta}}, \rho \in \Gamma$ a set $M_{\rho}$ such that the invariant measure $\mu_{f}$ is absolutely continuous w.r.t. Lebesgue measure $l$ if $\mu_{f}\left(\left[b_{1}, b_{2}\right]\right) \in M_{\rho}$.

We will show that this assumption contradicts Proposition 1.

For this, we derive first some properties of the distribution of $\log D h_{\bar{\theta}}^{q_{n}}(x)$ with respect to the partition $\mathcal{P}_{n}\left(h_{\bar{\theta}}\right)$ of Herman's $P L$-homeomorphisms $h_{\bar{\theta}}$ defined in Theorem 11.

For this, recall the sets $A_{n}\left(i_{0}\right), B_{n}\left(i_{0}\right)$ defined in (5.3), (5.6), respectively, (5.15). Later we will need also the closely related sets $A_{n}^{*}\left(i_{0}\right), B_{n}^{*}\left(i_{0}\right)$, defined according to Lemmas $3-5$ as follows:

- if the break point $c_{0}$ of $D h^{q_{n}}$ fulfills the assumption in Lemmas 3 or 5 , we set (see Fig. 2 and Fig. 4)

$$
A_{n}^{*}\left(i_{0}\right):=\bigcup_{s=1}^{i_{0}}\left[a_{-q_{n}+s}^{*}, a_{q_{n-1}+s}\right]
$$

and

$$
B_{n}^{*}\left(i_{0}\right):=\bigcup_{s=i_{0}+1}^{q_{n}}\left[a_{-q_{n}+s}^{*}, a_{q_{n-1}+s}\right]
$$

- if the break point $c_{0}$ of $D h^{q_{n}}$, on the other hand, fulfills the assumption in Lemma 4, we set (see Fig. 3)

$$
A_{n}^{*}\left(i_{0}\right):=\bigcup_{s=1}^{i_{0}}\left[a_{-q_{n}+s}^{*}, c_{-i_{0}+q_{n-1}+s}\right]
$$

and

$$
B_{n}^{*}\left(i_{0}\right):=\bigcup_{s=1}^{q_{n}-i_{0}-1}\left[c_{-q_{n}+s}^{*}, c_{q_{n-1}+s}\right]
$$

Consider first the case when the break point $c_{0}$ of $D h^{q_{n}}$ fulfills the assumption of Lemma 3 or Lemma 4. We begin our discussion with the case of Lemma 3. By Theorem 10

$$
\frac{1}{\log \sigma} \log D h^{q_{n}}(x)=(-1)^{n}\left(\mu_{\bar{\theta}}\left(A_{n}\left(i_{0}\right)\right)+\mu_{\bar{\theta}}\left(B_{n}\left(i_{0}\right)\right)\right)=q_{n} \cdot \frac{\beta}{1+\beta} \quad \bmod 1
$$

for all $x \in A_{n}\left(i_{0}\right) \cup B_{n}\left(i_{0}\right)$ as defined in (5.3). Therefore,

$$
\frac{1}{\log \sigma} \log D h^{q_{n}}(x) \quad \bmod 1= \begin{cases}1-\left(\mu_{\bar{\theta}}\left(A_{n}\left(i_{0}\right)\right)+\mu_{\bar{\theta}}\left(B_{n}\left(i_{0}\right)\right)\right) & \text { if } n \text { is odd } \\ \mu_{\bar{\theta}}\left(A_{n}\left(i_{0}\right)\right)+\mu_{\bar{\theta}}\left(B_{n}\left(i_{0}\right)\right) & \text { if } n \text { is even. }\end{cases}
$$

Next, we estimate $\mu_{\bar{\theta}}\left(A_{n}\left(i_{0}\right)\right)+\mu_{\bar{\theta}}\left(B_{n}\left(i_{0}\right)\right)$. By Lemma 3 (see Fig. 2) we have obviously

$$
A_{n}\left(i_{0}\right)=\bigcup_{s=1}^{i_{0}}\left[c_{-i_{0}+s}^{*}, a_{-q_{n}+s}^{*}\right] \subset \bigcup_{s=1}^{i_{0}}\left[a_{q_{n}+s}, a_{-q_{n}+s}^{*}\right]
$$

and

$$
B_{n}\left(i_{0}\right)=\bigcup_{s=i_{0}+1}^{q_{n}}\left[c_{-i_{0}-q_{n}+s}^{*}, a_{-q_{n}+s}^{*}\right] \subset \bigcup_{s=i_{0}+1}^{q_{n}}\left[a_{s}, a_{-q_{n}+s}^{*}\right] .
$$


It is easy to see that

$$
\mu_{\bar{\theta}}\left(A_{n}\left(i_{0}\right)\right) \leqslant 2 i_{0} \cdot \Delta_{n}, \quad \mu_{\bar{\theta}}\left(B_{n}\left(i_{0}\right)\right) \leqslant\left(q_{n}-i_{0}\right) \cdot \Delta_{n},
$$

Using these bounds and the recurrence relations

$$
q_{n}=k_{n} q_{n-1}+q_{n-2}, \quad \Delta_{n}=k_{n} \Delta_{n+1}+\Delta_{n+2}, n \geqslant 1,
$$

we obtain

$$
\begin{gathered}
\mu_{\bar{\theta}}\left(A_{n}\left(i_{0}\right)\right)+\mu_{\bar{\theta}}\left(B_{n}\left(i_{0}\right)\right) \leqslant\left(q_{n}+i_{0}\right) \cdot \Delta_{n} \leqslant \frac{\left(q_{n}+q_{n-1}\right) \Delta_{n}}{q_{n} \Delta_{n-1}}= \\
=\left(1+\frac{q_{n-1}}{q_{n}}\right) \cdot \frac{\Delta_{n}}{\Delta_{n-1}}<\left(1+\frac{1}{k_{n}}\right) \cdot \frac{1}{k_{n+1}} .
\end{gathered}
$$

Since $k_{n} \geqslant 3$ for $n \geqslant N_{\rho}$, we hence get

$$
\mu_{\bar{\theta}}\left(A_{n}\left(i_{0}\right)\right)+\mu_{\bar{\theta}}\left(B_{n}\left(i_{0}\right)\right)<\frac{4}{9}
$$

for $n \geqslant N_{\rho}$. Summarizing Eqs. (6.17), (6.18) and the last bound, we conclude that in the case of Lemma 3 and $n>N_{\rho}$

$$
0 \leqslant \frac{1}{\log \sigma} \log D h_{\bar{\theta}}^{q_{n}}(x)(\bmod 1)<\frac{4}{9}, \text { for } n \text { even, }
$$

and

$$
\frac{5}{9}<\frac{1}{\log \sigma} \log D h_{\frac{q_{n}}{\theta}}^{q}(x)(\bmod 1) \leqslant 1, \quad \text { for } n \text { odd. }
$$

Consider now the case of Lemma 4.

Using the second assertion of Theorem 10, it follows that

$$
\frac{1}{\log \sigma} \log D h_{\bar{\theta}}^{q_{n}}(x)(\bmod 1) \in\left\{\left|\mu_{\bar{\theta}}\left(B_{n}\left(i_{0}\right)\right)-\mu_{\bar{\theta}}\left(A_{n}\left(i_{0}\right)\right)\right|, 1-\left|\mu_{\bar{\theta}}\left(B_{n}\left(i_{0}\right)\right)-\mu_{\bar{\theta}}\left(A_{n}\left(i_{0}\right)\right)\right|\right\}
$$

for all $x \in A_{n}\left(i_{0}\right) \cup B_{n}\left(i_{0}\right)$ defined in (5.15). Lemma 4 and (5.15) imply for $n \geqslant N_{\rho}$

$$
\begin{gathered}
\left|\mu_{\bar{\theta}}\left(A_{n}\left(i_{0}\right)\right)-\mu_{\bar{\theta}}\left(B_{n}\left(i_{0}\right)\right)\right| \leqslant \mu_{\bar{\theta}}\left(A_{n}\left(i_{0}\right)\right)+\mu_{\bar{\theta}}\left(B_{n}\left(i_{0}\right)\right)< \\
<i_{0} \cdot \Delta_{n}+\left(q_{n}-i_{0}\right) \cdot \Delta_{n}=q_{n} \cdot \Delta_{n}<\frac{q_{n} \cdot \Delta_{n}}{q_{n} \cdot \Delta_{n-1}}=\frac{\Delta_{n}}{\Delta_{n-1}}<\frac{1}{3} .
\end{gathered}
$$

This and (6.25) lead, in case the break point $c_{0}$ of $D h^{q_{n}}$ fulfills the assumption of Lemma 4, to

$$
\frac{1}{\log \sigma} \log D h_{\frac{q_{n}}{\theta}}(x)(\bmod 1) \in\left[0, \frac{1}{3}\right] \cup\left[\frac{2}{3}, 1\right] .
$$

This together with the bounds (6.23) and (6.24) show that, in case the break point $c_{0}$ of $D h^{q_{n}}$ fulfills either the assumption of Lemma 3 or of Lemma $4, \frac{1}{\log \sigma} \log D h_{\bar{\theta}}^{q_{n}}(x)(\bmod 1)$ takes values only in $[0,1] \backslash\left[\frac{4}{9}, \frac{5}{9}\right]$, i.e., for $n \geqslant N_{\rho}$ and $x \in A_{n}\left(i_{0}\right) \cup B_{n}\left(i_{0}\right)$

$$
\frac{1}{\log \sigma} \log D h_{\bar{\theta}}^{q_{n}}(x) \quad \bmod 1 \in\left[0, \frac{4}{9}\right] \cup\left[\frac{5}{9}, 1\right] .
$$


From Theorem 10, however, we know that the sequence

$$
\left\{\frac{1}{\log \sigma} \log D h_{\frac{q_{n}}{\theta}}(x)=q_{n} \cdot \frac{\beta}{1+\beta} \bmod 1\right\}
$$

is uniformly distributed on $[0,1]$ and hence its values are dense in $[0,1]$. From $(6.26)$ we therefore conclude that the cases where the break point $c_{0}$ of $D h^{q_{n}}$ fulfills either the assumption of Lemma 3 or of Lemma 4 can happen only for a finite number of $q_{n}$ 's. This means, on the other hand, that the values of $\frac{1}{\log \sigma} \log D h_{\bar{\theta}}^{q_{n}}$ are dense on [0,1] only in case the break point $c_{0}$ of $D h_{\bar{\theta}}^{q_{n}}$ fulfills the assumption of Lemma 5 .

Hence, we have to discuss next this case. By Theorem 10 we have for $x \in A_{n}\left(i_{0}\right) \cup B_{n}\left(i_{0}\right)$, defined in (5.6),

$$
\frac{1}{\log \sigma} \log D h^{q_{n}}(x)=(-1)^{n}\left(\mu_{\bar{\theta}}\left(A_{n}\left(i_{0}\right)\right)+\mu_{\bar{\theta}}\left(B_{n}\left(i_{0}\right)\right)\right) \quad \bmod 1 .
$$

But this means that

$$
D h^{q_{n}}(x) \quad \bmod 1= \begin{cases}\mu_{\bar{\theta}}\left(A_{n}\left(i_{0}\right)\right)+\mu_{\bar{\theta}}\left(B_{n}\left(i_{0}\right)\right) & \text { if } n \text { is even } \\ 1-\left(\mu_{\bar{\theta}}\left(A_{n}\left(i_{0}\right)\right)+\mu_{\bar{\theta}}\left(B_{n}\left(i_{0}\right)\right)\right) & \text { if } n \text { is odd }\end{cases}
$$

We can assume that for some subsequence $\left\{q_{n_{m}}\right\}$

$$
\lim _{m \rightarrow \infty} D h^{q_{2 n_{m}}}(x)=\lim _{m \rightarrow \infty}\left(\mu_{h}\left(B_{2 n_{m}}\left(i_{0}\right)\right)+\mu_{h}\left(A_{n_{2 n_{m}}}\left(i_{0}\right)\right)\right)=\omega
$$

with $\omega \in[0,1]$. The case

$$
\lim _{m \rightarrow \infty} D h^{q_{2 n_{m}+1}}(x)=\lim _{m \rightarrow \infty}\left(1-\left(\mu_{h}\left(B_{2 n_{m}+1}\left(i_{0}\right)\right)+\mu_{h}\left(A_{n_{2 n_{m}+1}}\left(i_{0}\right)\right)\right)\right)=\omega
$$

with $\omega \in[0,1]$ can be treated analogously.

We have to consider two possible cases, namely, when $0 \leqslant i_{0}<\frac{q_{n}}{2}$ and when $\frac{q_{n}}{2} \leqslant i_{0}<q_{n}$. Consider the case $0 \leqslant i_{0}<\frac{q_{n}}{2}$.

We start with several bounds and estimate first the ratio $\frac{\mu_{\bar{\theta}}\left(A_{n}\left(i_{0}\right)\right)}{\mu_{\bar{\theta}}\left(B_{n}\left(i_{0}\right)\right)}$. It is clear that $\frac{\mu_{\bar{\theta}}\left(A_{n}\left(i_{0}\right)\right)}{\mu_{\bar{\theta}}\left(B_{n}\left(i_{0}\right)\right)}=\frac{i_{0} \mu_{\bar{\theta}}\left(\left[a_{-q_{n}+1}^{*}, c_{-i_{0}+1}^{*}\right]\right)}{\left(q_{n}-i_{0}\right) \mu_{\bar{\theta}}\left(\left[a_{-q_{n}+1}^{*}, c_{-i_{0}+1}^{*}\right]\right)+\left(q_{n}-i_{0}\right) \mu_{\bar{\theta}}\left(\left[c_{1}, c_{-q_{n}+1}^{*}\right]\right)} \leqslant \frac{i_{0}}{q_{n}-i_{0}} \leqslant \frac{\frac{q_{n}}{2}}{q_{n}-\frac{q_{n}}{2}}=1$, where we have used $0 \leqslant i_{0}<\frac{q_{n}}{2}$. Consequently,

$$
\mu_{\bar{\theta}}\left(A_{n}\left(i_{0}\right)\right) \leqslant \mu_{\bar{\theta}}\left(B_{n}\left(i_{0}\right)\right) .
$$

Using this bound, we obtain $1 \geqslant \mu_{\bar{\theta}}\left(A_{n}\left(i_{0}\right)\right)+\mu_{\bar{\theta}}\left(B_{n}\left(i_{0}\right)\right) \geqslant 2 \mu_{\bar{\theta}}\left(A_{n}\left(i_{0}\right)\right)$, which implies

$$
\mu_{\bar{\theta}}\left(A_{n}\left(i_{0}\right)\right) \leqslant \frac{1}{2}
$$


From the definition of $B_{n}\left(i_{0}\right)$ and $B_{n}^{*}\left(i_{0}\right)$ in (5.6) and (6.14) it follows that

$$
\mu_{\bar{\theta}}\left(B_{n}^{*}\left(i_{0}\right)\right)=\left(q_{n}-i_{0}\right) \Delta_{n-1}-\mu_{\bar{\theta}}\left(B_{n}\left(i_{0}\right)\right) .
$$

Next, we estimate $\left(q_{n}-i_{0}\right) \Delta_{n-1}$. It is obvious that

$$
q_{n} \Delta_{n-1}+q_{n-1} \Delta_{n}=1 .
$$

It is clear that

$$
\frac{q_{n} \Delta_{n-1}}{q_{n-1} \Delta_{n}}=\frac{k_{n} q_{n-1}+q_{n-2}}{q_{n-1}} \cdot \frac{k_{n+1} \Delta_{n}+\Delta_{n+2}}{\Delta_{n}}=\left(k_{n}+\frac{q_{n-2}}{q_{n-1}}\right)\left(k_{n+1}+\frac{\Delta_{n+1}}{\Delta_{n}}\right) .
$$

This and (6.33) imply

$$
q_{n} \Delta_{n-1}>1-\frac{1}{k_{n} k_{n+1}+1}=\frac{k_{n} k_{n+1}}{k_{n} k_{n+1}+1} .
$$

Next, we compare $q_{n} \Delta_{n-1}$ and $\left(q_{n}-i_{0}\right) \Delta_{n-1}$. Since $0 \leqslant i_{0}<\frac{q_{n}}{2}$, we have

$$
\frac{\left(q_{n}-i_{0}\right) \Delta_{n-1}}{q_{n} \Delta_{n-1}} \geqslant \frac{1}{2}
$$

Together with (6.34) this implies that

$$
\left(q_{n}-i_{0}\right) \Delta_{n-1} \geqslant \frac{1}{2} q_{n} \Delta_{n-1} \geqslant \frac{1}{2} \cdot \frac{k_{n} k_{n+1}}{k_{n} k_{n+1}+1} .
$$

Since $k_{n} \geqslant 3$ for $n \geqslant N_{\rho}$, we therefore have shown

$$
\left(q_{n}-i_{0}\right) \Delta_{n-1} \geqslant \frac{1}{2} \cdot \frac{9}{10}=\frac{9}{20} .
$$

Suppose now $n_{m}, m=1,2, \ldots$ is some sequence of even numbers such that

$$
\lim _{m \rightarrow \infty}\left(\mu_{\bar{\theta}}\left(B_{n_{m}}\left(i_{0}\right)\right)+\mu_{\bar{\theta}}\left(A_{n_{m}}\left(i_{0}\right)\right)\right)=\omega_{1}
$$

with $\omega_{1} \in[0,1]$. Then, for any $\varepsilon>0$ and sufficiently large $n_{m}$,

$$
\omega_{1}-\mu_{\bar{\theta}}\left(A_{n_{m}}\left(i_{0}\right)\right)-\varepsilon \leqslant \mu_{\bar{\theta}}\left(B_{n_{m}}\left(i_{0}\right)\right) \leqslant \omega_{1}-\mu_{\bar{\theta}}\left(A_{n_{m}}\left(i_{0}\right)\right)+\varepsilon .
$$

This together with (6.31) implies

$$
\omega_{1}-\frac{1}{2}-\epsilon \leqslant \mu_{\bar{\theta}}\left(B_{n}\left(i_{0}\right)\right)<\omega_{1}+\epsilon .
$$

This together with (6.32) implies for sufficiently large $n_{m}$

$$
\mu_{\bar{\theta}}\left(B_{n_{m}}^{*}\left(i_{0}\right)\right) \geqslant \frac{9}{20}-\omega_{1}-\varepsilon
$$

The last two relations show that $\mu_{\bar{\theta}}\left(B_{n_{m}}\left(i_{0}\right)\right)$ and $\mu_{\bar{\theta}}\left(B_{n_{m}}^{*}\left(i_{0}\right)\right)$ are comparable with some constant $C>1$. 
In the next step of the proof of Theorem 3 we consider two copies of the unit circle. Suppose the homeomorphism $f$ acts on the first circle, and Herman's homeomorphism $h_{\bar{\theta}}$, on the second one. It is obvious that

$$
\mu_{f}\left(\varphi^{-1}\left(B_{n_{m}}\left(i_{0}\right)\right)\right)=\mu_{\bar{\theta}}\left(B_{n_{m}}\left(i_{0}\right)\right), \quad \mu_{f}\left(\varphi^{-1}\left(B_{n_{m}}^{*}\left(i_{0}\right)\right)\right)=\mu_{\bar{\theta}}\left(B_{n_{m}}^{*}\left(i_{0}\right)\right) .
$$

These relations together with (6.37) imply that

$$
c_{1} \leqslant \mu_{f}\left(\varphi^{-1}\left(A_{n_{m}}\left(i_{0}\right)\right)\right) \leqslant c_{2}, \quad c_{1} \leqslant \mu_{f}\left(\varphi^{-1}\left(A_{n_{m}}^{*}\left(i_{0}\right)\right)\right) \leqslant c_{2} .
$$

The last relations, the arrangement of the break points of $f^{q_{n}}$ and absolutely continuity of the invariant measure $\mu_{f}$ w.r.t. Lebesgue measure $l$ imply for sufficiently large $n_{m}$ the following bounds:

$$
c_{3} \leqslant l\left(\varphi^{-1}\left(A_{n_{m}}\left(i_{0}\right)\right)\right) \leqslant c_{4}, \quad c_{3} \leqslant l\left(\varphi^{-1}\left(A_{n_{m}}^{*}\left(i_{0}\right)\right)\right) \leqslant c_{4}
$$

for some constants $c_{3}, c_{4} \in(0,1)$.

Consider next the set of break points

$$
B P_{1}\left(f^{q_{n_{m}}}\right):=\left\{c_{-q_{n}+1}^{*}, c_{-q_{n}+2}^{*}, . ., c_{-i_{0}}^{*}\right\} \subset B P\left(f^{q_{n_{m}}}, c_{-i_{0}}^{*}\right)
$$

and for $z \in B P_{1}\left(f^{q_{n_{m}}}\right)$ the left and right normalized $\delta$-neighborhoods $V_{n_{m}}^{l}(z, \delta)$, respectively, $V_{n_{m}}^{r}(z, \delta)$. Put

$$
V_{n_{m}}^{l}(\delta):=\bigcup_{z \in B P_{1}\left(f^{q_{n_{m}}}\right)} V_{n}^{l}(z, \delta), \quad V_{n_{m}}^{r}(\delta):=\bigcup_{z \in B P_{1}\left(f^{q_{n_{m}}}\right)} V_{n_{m}}^{r}(z, \delta) .
$$

Obviously,

$$
V_{n_{m}}^{l}(\delta) \subset \varphi^{-1}\left(B_{n_{m}}\left(i_{0}\right)\right), \quad V_{n_{m}}^{r}(\delta) \subset \varphi^{-1}\left(B_{n_{m}}^{*}\left(i_{0}\right)\right) .
$$

We conclude that the length $l$ of each of these intervals covering the normalized $\delta$-neighborhoods by definition is $\delta^{-1}$ times the length of the latter ones. Using the definitions of $B_{n_{m}}\left(i_{0}\right), B_{n_{m}}^{*}\left(i_{0}\right)$ (see also Fig. 4 ) and the normalized one-sided $\delta$-neighborhoods $V_{n}^{\cdot}(z, \delta)$, we obtain

$$
\begin{aligned}
& l\left(\varphi^{-1}\left(B_{n_{m}}\left(i_{0}\right) \cap V_{n_{m}}^{l}(\delta)\right)\right)=\delta \cdot l\left(\varphi^{-1}\left(B_{n_{m}}\left(i_{0}\right)\right)\right), \\
& l\left(\varphi^{-1}\left(B_{n_{m}}^{*}\left(i_{0}\right) \cap V_{n_{m}}^{r}(\delta)\right)\right)=\delta \cdot l\left(\varphi^{-1}\left(B_{n_{m}}^{*}\left(i_{0}\right)\right)\right)
\end{aligned}
$$

and consequently

$$
l\left(\left(\varphi^{-1}\left(B_{n_{m}}\left(i_{0}\right)\right) \cup \varphi^{-1}\left(B_{n_{m}}^{*}\left(i_{0}\right)\right)\right) \cap\left(V_{n_{m}}^{l}(\delta) \cup V_{n_{m}}^{r}(\delta)\right)\right)=\delta \cdot l\left(\varphi^{-1}\left(B_{n_{m}}\left(i_{0}\right) \cup B_{n_{m}}^{*}\left(i_{0}\right)\right)\right) .
$$

From this we can now derive bounds on the values of $D f^{q_{n_{m}}}$. Put $\delta=\left|\frac{\log \Lambda}{a K e^{v}}\right|$ with $\Lambda$ as defined in Lemma 7 and $a>\left|\frac{\log \Lambda}{K e^{v}}\right|$. From relations (6.3) and (6.4) of Lemma 7 it follows that either on the left or on the right normalized $\delta$-neighborhood of every break point $z$ of $f^{q_{n_{m}}}$

$$
\left|\log D f^{q_{n_{m}}}(x)\right| \geqslant\left|\frac{(a-2) \log \Lambda}{2 a}\right| .
$$

Define

$$
G_{n_{m}}(\delta)=\bigcup\left\{z \in B P_{1}\left(f^{q_{n_{m}}}\right):\left|\log D f^{q_{n}}(x)\right| \geqslant\left|\frac{(a-2) \log \Lambda}{2 a}\right| \text { for all } x \in V_{n_{m}}^{s}(z, \delta), s \in\{l, r\}\right\} .
$$


It is clear that

$$
l\left(G_{n_{m}}(\delta)\right) \geqslant \min \left\{\delta \cdot l\left(B_{n_{m}}\left(i_{0}\right)\right), \quad \delta \cdot l\left(B_{n_{m}}^{*}\left(i_{0}\right)\right)\right\} \geqslant c_{5} \cdot \delta
$$

with some constant $c_{5}>0$. Finally, we obtain

$$
\left|\log D f^{q_{n_{m}}}(x)\right| \geqslant\left|\frac{(a-2) \log \Lambda}{2 a}\right|
$$

for all $x \in G_{n_{m}}(\delta)$. But this contradicts convergence of $D f^{q_{n}(x)}$ to 1 in probability with respect to normalized Lebesgue measure.

In the second case when $\frac{q_{n}}{2} \leqslant i_{0}<q_{n}$ we will show only that either $A_{n}\left(i_{0}\right)$ and $A_{n}^{*}\left(i_{0}\right)$ or $B_{n}\left(i_{0}\right)$ and $B_{n}^{*}\left(i_{0}\right)$ are comparable. The construction of the sets $G_{n_{m}}$ in these two cases proceeds in complete analogy to the case when $0 \leqslant i_{0}<\frac{q_{n}}{2}$.

In the case $\frac{q_{n}}{2} \leqslant i_{0}<q_{n}$ assume first

$$
\mu_{\bar{\theta}}\left(A_{n}\left(i_{0}\right)\right) \geqslant \mu_{\bar{\theta}}\left(B_{n}\left(i_{0}\right)\right) .
$$

We will then show that $\mu_{\bar{\theta}}\left(A_{n_{m}}\left(i_{0}\right)\right)$ and $\mu_{\bar{\theta}}\left(A_{n_{m}}^{*}\left(i_{0}\right)\right)$ are comparable for some subsequence $\left\{n_{m}\right\}$. Using the definition of $A_{n}\left(i_{0}\right)$ and $A_{n}^{*}\left(i_{0}\right)$ and Lemma 5 , we get

$$
\mu_{\bar{\theta}}\left(A_{n}^{*}\left(i_{0}\right)\right)=i_{0} \Delta_{n-1}-\mu_{\bar{\theta}}\left(A_{n}\left(i_{0}\right)\right) .
$$

Assume again we are given a sequence $n_{m}, m=1,2, \ldots$ of even numbers such that

$$
\lim _{m \rightarrow \infty}\left(\mu_{\bar{\theta}}\left(B_{n_{m}}\left(i_{0}\right)\right)+\mu_{\bar{\theta}}\left(A_{n_{m}}\left(i_{0}\right)\right)\right)=\omega_{2}
$$

with $\omega_{2} \in[0,1]$. Then for any $\epsilon>0$ and for sufficiently large $n_{m}$

$$
\omega_{2}-\epsilon<\mu_{\bar{\theta}}\left(A_{n_{m}}\left(i_{0}\right)\right)+\mu_{\bar{\theta}}\left(B_{n_{m}}\left(i_{0}\right)\right)<\omega_{2}+\epsilon .
$$

This together with (6.43) implies

$$
\frac{\omega_{2}-\epsilon}{2}<\mu_{\bar{\theta}}\left(A_{n_{m}}\left(i_{0}\right)\right)<\omega_{2}+\epsilon
$$

Hence, together with (6.35) and (6.43) we get

$$
\begin{aligned}
\mu_{\bar{\theta}}\left(A_{n_{m}}^{*}\left(i_{0}\right)\right) & =i_{0} \Delta_{n_{m}-1}-\mu_{\bar{\theta}}\left(A_{n_{m}}\left(i_{0}\right)\right) \geqslant \\
& \geqslant \frac{1}{2} q_{n_{m}} \Delta_{n_{m}-1}-\omega_{2}-\epsilon \geqslant \frac{1}{2} \cdot \frac{9}{20}-\omega_{2}-\epsilon=\frac{9}{40}-\omega_{2}-\epsilon .
\end{aligned}
$$

The bounds (6.47) and (6.48) show that, choosing $\omega_{2}$ less than $\frac{9}{40}$, one finds for sufficiently large $n$ and $\epsilon>0$ small

$$
\mu_{\bar{\theta}}\left(A_{n_{m}}\left(i_{0}\right)\right)>c_{1} \quad \text { and } \quad \mu_{\bar{\theta}}\left(A_{n_{m}}^{*}\left(i_{0}\right)\right)>c_{2}
$$

for some constants $c_{1}, c_{2}>0$. Hence, under the assumptions $(6.43) \mu_{\bar{\theta}}\left(A_{n_{m}}\left(i_{0}\right)\right)$ and $\mu_{\bar{\theta}}\left(A_{n_{m}}^{*}\left(i_{0}\right)\right)$ are comparable with some constant $K>1$. 
Assume next in the case $\frac{q_{n}}{2} \leqslant i_{0}<q_{n}$

$$
\mu_{\bar{\theta}}\left(A_{n}\left(i_{0}\right)\right)<\mu_{\bar{\theta}}\left(B_{n}\left(i_{0}\right)\right) .
$$

This assumption and (6.46) imply:

$$
\frac{\omega_{2}-\epsilon}{2}<\mu_{\bar{\theta}}\left(B_{n_{m}}\left(i_{0}\right)\right)<\omega_{2}+\epsilon .
$$

Using the definition of $A_{n}\left(i_{0}\right), B_{n}\left(i_{0}\right)$, Eq. (6.44) and Lemma 5 (see Fig. 4), we obtain

$$
\omega_{2}-\epsilon<\mu_{\bar{\theta}}\left(A_{n_{m}}\left(i_{0}\right)\right)+\mu_{\bar{\theta}}\left(B_{n_{m}}\left(i_{0}\right)\right)=q_{n} \mu_{\bar{\theta}}\left(\left[a_{-q_{n_{m}}+1}^{*}, c_{-i_{0}+1}^{*}\right]\right)+\left(q_{n_{m}}-i_{0}\right) \Delta_{n_{m}}<\omega_{2}+\epsilon .
$$

Put $i_{0}:=i_{0}\left(n_{m}\right)=l_{0}\left(n_{m}\right) q_{n_{m}}, 0 \leqslant l_{0}\left(n_{m}\right) \leqslant 1$. Then we have

$$
\left(q_{n_{m}}-i_{0}\right) \Delta_{n_{m}}=\left(1-l_{0}\right) q_{n_{m}} \cdot \Delta_{n_{m}} .
$$

It easy to show that

$$
\frac{9}{40}<q_{n_{m}} \Delta_{n_{m}}<\frac{1}{3}
$$

and hence

$$
\frac{9}{40}\left(1-l_{0}\right)<\left(q_{n_{m}}-i_{0}\right) \Delta_{n_{m}}<\frac{1}{3}\left(1-l_{0}\right)
$$

Assume now

$$
\frac{1}{3}\left(1-l_{0}\right)<\frac{\omega_{2}-\epsilon}{2},
$$

then by (6.52) and (6.54) we obtain

$$
q_{n_{m}} \mu_{\bar{\theta}}\left(\left[a_{-q_{n_{m}}+1}^{*}, c_{-i_{0}+1}^{*}\right]\right)>\frac{\omega_{2}-\epsilon}{2},
$$

which together with $(6.52)$ and $q_{n_{m}}-i_{0}<i_{0}$ implies

$$
\begin{aligned}
\frac{\omega_{2}-\epsilon}{2} & <q_{n_{m}} \mu_{\bar{\theta}}\left(\left[a_{-q_{n_{m}}+1}^{*}, c_{-i_{0}+1}^{*}\right]\right)= \\
& =i_{0} \mu_{\bar{\theta}}\left(\left[a_{-q_{n_{m}+1}}^{*}, c_{-i_{0}+1}^{*}\right]\right)+\left(q_{n_{m}}-i_{0}\right) \mu_{\bar{\theta}}\left(\left[a_{-q_{n_{m}}+1}^{*}, c_{-i_{0}+1}^{*}\right]\right) \leqslant \\
& \leqslant 2 i_{0} \mu_{\bar{\theta}}\left(\left[a_{-q_{n_{m}}+1}^{*}, c_{-i_{0}+1}^{*}\right]\right)=2 \mu_{\bar{\theta}}\left(A_{n_{m}}\left(i_{0}\right)\right)
\end{aligned}
$$

or

$$
\frac{\omega_{2}-\epsilon}{4}<\mu_{\bar{\theta}}\left(A_{n_{m}}\left(i_{0}\right)\right)
$$

Using this and (6.44), we can see that

$$
\mu_{\bar{\theta}}\left(A_{n_{m}}^{*}\left(i_{0}\right)\right) \geqslant \frac{9}{20}-\frac{\omega_{2}-\epsilon}{4}=\frac{9}{20}-\frac{\omega_{2}}{4}+\frac{\epsilon}{4},
$$

which together with (6.56) shows that, under assumptions $(6.55), \mu_{\bar{\theta}}\left(A_{n_{m}}\left(i_{0}\right)\right)$ and $\mu_{\bar{\theta}}\left(A_{n_{m}}^{*}\left(i_{0}\right)\right)$ are comparable for sufficiently large $n_{m}$ and sufficiently small $\epsilon>0$.

It remains to discuss the case where

$$
\frac{1}{3}\left(1-l_{0}\right) \geqslant \frac{\omega_{2}-\epsilon}{2}
$$


In this case we obtain, using $q_{n} \Delta_{n-1} \in\left(\frac{9}{10}, 1\right)$,

$$
\left(q_{n_{m}}-i_{0}\right) \Delta_{n_{m}}=\left(1-l_{0}\right) q_{n_{m}} \Delta_{n_{m}} \geqslant \frac{3}{2}\left(\omega_{2}-\epsilon\right) q_{n_{m}} \Delta_{n_{m}} \geqslant \frac{27}{20}\left(\omega_{2}-\epsilon\right) .
$$

Together with (6.51) we then get

$$
\mu_{\bar{\theta}}\left(B_{n_{m}}^{*}\left(i_{0}\right)\right)=\left(q_{n_{m}}-i_{0}\right) \Delta_{n_{m}-1}-\mu_{\bar{\theta}}\left(B_{n_{m}}\left(i_{0}\right)\right) \geqslant \frac{27}{20}\left(\omega_{2}-\epsilon\right)-\left(\omega_{2}+\epsilon\right) \geqslant \frac{7}{20} \omega_{2}-\frac{47}{20} \epsilon
$$

which together with (6.51) shows that, under the assumptions (6.50) and (6.57) for sufficiently large $n_{m}, \mu_{\bar{\theta}}\left(B_{n_{m}}\left(i_{0}\right)\right)$ and $\mu_{\bar{\theta}}\left(B_{n_{m}}^{*}\left(i_{0}\right)\right)$ are comparable if $\omega_{2}$ is small and $\epsilon$ is sufficiently small.

Finally, we conclude that, under the assumptions of Theorem 3, either $\mu_{\bar{\theta}}\left(B_{n_{m}}\left(i_{0}\right)\right)$ and $\mu_{\bar{\theta}}\left(B_{n_{m}}^{*}\left(i_{0}\right)\right)$ are K-comparable or $\mu_{\bar{\theta}}\left(A_{n_{m}}\left(i_{0}\right)\right)$ and $\mu_{\bar{\theta}}\left(A_{n_{m}}^{*}\left(i_{0}\right)\right)$ are K-comparable, with some constant $K>1$, depending on $\omega$.

\section{Acknowledgments}

This work was partially performed during the first author's (A. D.) visit in 2016 as a senior associate of ICTP, Italy, and a visit in 2018 to the Institute of Theoretical Physics of Clausthal University, supported by the German Academic Exchange Service DAAD and the Friends of Clausthal University. A. D. wants to thank K. Khanin for a very fruitful discussion and the Institute for Theoretical Physics and its director P. Blöchl for the kind hospitality.

\section{References}

[1] Adouani, A. and Marzougui, H., Singular Measures for Class P-Circle Homeomorphisms with Several Break Points, Ergodic Theory Dynam. Systems, 2014, vol. 34, no. 2, pp. 423-456.

[2] Arnol'd, V.I., Small Denominators: 1. Mapping the Circle onto Itself, Izv. Akad. Nauk SSSR. Ser. Mat., 1961, vol. 1, no. 1, pp. 21-86 (Russian).

[3] Coelho, Z., Lopes, A., and da Rocha, L.F., Absolutely Continuous Invariant Measures for a Class of Affine Interval Exchange Maps, Proc. Amer. Math. Soc., 1995, vol. 123, no. 11, pp. 3533-3542.

[4] Cornfeld, I. P., Fomin, S. V., and Sinai, Ya. G., Ergodic Theory, Grundlehren Math. Wiss., vol. 245, New York: Springer, 1982.

[5] Denjoy, A., Sur les courbes définies par les équations différentielles à la surface du tore, J. Math. Pures Appl. (9), 1932, vol.11, pp. 333-375.

[6] Dzhalilov, A. A. and Khanin, K. M., On an Invariant Measure for Homeomorphisms of a Circle with a Point of Break, Funct. Anal. Appl., 1998, vol.32, no. 3, pp. 153-161; see also: Funktsional. Anal. i Prilozhen., 1998, vol. 32, no. 3, pp. 11-21.

[7] Dzhalilov, A. A., Piecewise Smoothness of Conjugate Homeomorphisms of a Circle with Corners, Theoret. and Math. Phys., 1999, vol.120, no.2, pp.179-192; see also: Teoret. Mat. Fiz., 1999, vol. 120, no. 2, pp. 961-972.

[8] Dzhalilov, A. A. and Liousse, I., Circle Homeomorphisms with Two Break Points, Nonlinearity, 2006 , vol. 19, no. 8, pp. 1951-1968.

[9] Dzhalilov, A., Liousse, I., and Mayer, D., Singular Measures of Piecewise Smooth Circle Homeomorphisms with Two Break Points, Discrete Contin. Dyn. Syst., 2009, vol. 24, no. 2, pp. 381-403.

[10] Dzhalilov, A., Jalilov, A., and Mayer, D., A Remark on Denjoy's Inequality for PL Circle Homeomorphisms with Two Break Points, J. Math. Anal. Appl., 2018, vol.458, no. 1, pp. 508-520. 
[11] Dzhalilov, A. A., Mă̌er, D., and Safarov, U. A., Piecewise-Smooth Circle Homeomorphisms with Several Break Points, Izv. Math., 2012, vol.76, no. 1, pp.94-112; see also: Izv. Ross. Akad. Nauk Ser. Mat., 2012, vol. 76, no. 1, pp. 101-120.

[12] Katznelson, Y. and Ornstein, D., The Absolute Continuity of the Conjugation of Certain Diffeomorphisms of the Circle, Ergodic Theory Dynam. Systems, 1989, vol. 9, no. 4, pp. 681-690.

[13] Hawkins, J. and Schmidt, K., On $C^{2}$-Diffeomorphisms of the Circle Which Are of Type III ${ }_{1}$, Invent. Math., 1982, vol. 66, no. 3, pp. 511-518.

[14] Herman, M., Sur la conjugaison différentiable des difféomorphismes du cercle à des rotations, Inst. Hautes Études Sci. Publ. Math., 1979, vol. 49, pp. 5-233.

[15] Khanin, K. M. and Kocić, S., Hausdorff Dimension of Invariant Measure of Circle Diffeomorphisms with Breaks, Ergod. Theory Dyn. Syst., 2017, vol.63, pp.1-9.

[16] Sinai, Ya. G. and Khanin, K. M., Smoothness of Conjugacies of Diffeomorphisms of the Circle with Rotations, Russian Math. Surveys, 1989, vol.44, no. 1, pp. 69-99; see also: Uspekhi Mat. Nauk, 1989, vol. 44, no. 1(265), pp. 57-82, 247.

[17] Kuipers, L. and Niederreiter, H., Uniform Distribution of Sequences, New York: Wiley, 1974.

[18] Larcher, G., A Convergence Problem Connected with Continued Fractions, Proc. Amer. Math. Soc., 1988, vol. 103, no. 3, pp. 718-722.

[19] Liousse, I., $P L$ Homeomorphisms of the Circle Which Are Piecewise $C^{1}$ Conjugate to Irrational Rotations, Bull. Braz. Math. Soc. (N.S.), 2004, vol. 35, no. 2, pp. 269-280.

[20] Liousse, I., Nombre de rotation, mesures invariantes et ratio set des homéomorphismes affines par morceaux du cercle, Ann. Inst. Fourier (Grenoble), 2005, vol.55, no. 2, pp. 431-482.

[21] Nakada, H., Piecewise Linear Homeomorphisms of Type III and the Ergodicity of Cylinder Flows, Keio Math. Sem. Rep., 1982, No.7, pp. 29-40.

[22] Teplinsky, A., A Circle Diffeomorphism with Breaks That Is Smoothly Linearizable, Ergod. Theory Dyn. Syst., 2018, vol.38, no. 1, pp. 371-383.

[23] Yoccoz, J.-Ch., Il n'y a pas de contre-exemple de Denjoy analytique, C. R. Acad. Sci. Paris Sér. 1 Math., 1984, vol. 298, no.7, pp. 141-144. 